\title{
Étude didactique de la dialectique du travail et de la formation au bloc opératoire
}

A didactical study of the work and training dialectic in the operating theatre

Lucile Vadcard

\section{(2) OpenEdition}

\section{Journals}

Édition électronique

URL : http://journals.openedition.org/educationdidactique/1598

DOI : 10.4000/educationdidactique.1598

ISSN : 2111-4838

Éditeur

Presses universitaires de Rennes

\section{Édition imprimée}

Date de publication : 7 février 2013

Pagination : 117-146

ISBN : 978-2-7535-2261-9

ISSN : 1956-3485

Référence électronique

Lucile Vadcard, «Étude didactique de la dialectique du travail et de la formation au bloc opératoire », Éducation et didactique [En ligne], 7-1 | 2013, mis en ligne le 31 janvier 2015, consulté le 01 mai 2019. URL : http://journals.openedition.org/educationdidactique/1598; DOI : 10.4000/ educationdidactique. 1598 


\title{
ÉTUDE DIDACTIQUE DE LA DIALECTIQUE DU TRAVAIL ET DE LA FORMATION AU BLOC OPÉRATOIRE
}

\author{
Lucile Vadcard \\ Laboratoire des Sciences de l'Éducation - Université Pierre-Mendès-France, Grenoble 2
}

Cet article présente une étude didactique de la formation professionnelle des internes au bloc opératoire. Une spécificité importante des interactions entre l'apprenti et le maître dans ce contexte est qu'elles se déroulent en situation de travail : les enjeux professionnels et les enjeux de formation doivent coexister. Nous centrons cette étude sur la description de la nature des interactions et de la nature des contenus sur lesquelles elles portent. Ces deux entrées nous permettent d'identifier un contrat didactique particulier, mais néanmoins proche de celui étudié en didactique des disciplines, et qui semble permettre la coexistence, dans un équilibre qui varie, de ces deux enjeux à première vue incompatibles.

Mots-clés : Compagnonnage, Contrat didactique, Internat, Formation professionnelle

\section{A didactical study of the work and training dialectic in the operating theatre}

This paper presents a didactical study of apprenticeship in operating theatre. One specific aspect of the interactions between the apprentice and the master in this context is that they occur during work: professional and training issues have to coexist. Our study describes the kind of interactions that occur and the kind of content they deal with. These two entries allow us to identify sort of a didactical contract, like the one which is studied in didactics of disciplines. This contract seems to allow the coexistence, in a variable balance, of the two previous mentioned issues, which are at first glance incompatible.

Keywords: Apprenticeship, Didactical contract, Internship, Professional learning 


\section{INTRODUCTION}

Cet article traite de formation au travail. Plus précisément, nous nous intéressons aux situations dans lesquelles les enjeux professionnels côtoient, intentionnellement, les enjeux de formation des novices. Ces situations sont reconnues efficaces dans certaines formations (nous pensons en particulier ici à l'internat, mais également aux écoles de Compagnons du Devoir, considérées comme l'élite des formations aux métiers de l'artisanat), mais plus questionnées dans d'autres formations, où elles se juxtaposent plus ou moins heureusement avec des dispositifs scolaires. C'est le cas des formations par alternance, comme celles des professions paramédicales (infirmière, kinésithérapeuthe, manipulateur d'électroradiologie médicale, etc.), et de certains dispositifs dits "d'apprentissage », souvent mis en place pour les métiers de bas niveau de qualification (métiers du bâtiment, artisanat).

Nous pensons que la didactique a intérêt à porter un regard sur ces situations pour comprendre quelques-uns des déterminants de leur fonctionnement. Débuter une telle analyse par des formations réputées satisfaisantes nous paraît une bonne voie d'abord. À ce titre deux terrains nous intéressent particulièrement, l'internat et le compagnonnage, qui apparaissent après étude n'être pas si éloignés l'un de l'autre (Peneff, 1997). Nous nous centrons dans ce texte sur l'internat.

Nous posons l'hypothèse qu'au-delà de la contingence de ces situations de travail au cours desquelles se rencontrent les formateurs et les élèves, il existe une organisation didactique qui rationalise en partie les relations que les protagonistes entretiennent avec les savoirs. Nous posons également l'hypothèse que cette rationalité sera spécifique de ce type de situations (formation sur le lieu du travail) en ce qu'elle doit faire avec les enjeux du travail. Notre objectif principal est d'identifier dans quelle mesure ces deux ambitions, former et travailler, s'ajustent au cours de la situation; il s'agit de comprendre la spécificité de la présence de l'enjeu de travail pour celui de la formation, et de caractériser les phénomènes d'enseignement et d'apprentissage qui s'y déroulent.

Nous nous attacherons dans un premier temps à montrer l'existence d'un vide didactique sur la question des phénomènes d'enseignement et d'apprentissage sur le lieu du travail. D'un côté, les études qui portent sur le bloc opératoire, ou plus largement sur la formation médicale, n'ont pas, à notre connaissance, pris le parti de comprendre les phénomènes de formation qui s'y déroulent du point de vue des savoirs. D'un autre côté, nous trouvons un ensemble de travaux sur la formation professionnelle, pour la plupart hors du domaine médical, qui traitent de questions relatives à l'accompagnement, au tutorat. Ces travaux, issus en partie des sciences de l'éducation, de la psychologie du travail et de l'ergonomie cognitive, considèrent les dispositifs mis en place, sans interroger leur spécificité au regard des contenus dont ils sont censés permettre la transmission.

Cette première partie aura montré tout l'intérêt qu'il peut y avoir, pour la formation professionnelle, à développer une compréhension des modes de transmission au travail qui permette d'affiner le discours accompagnant généralement ces situations, dans lequel on trouve convoquées des notions telles que l'observation, l'imitation, l'entraînement, sans pour autant qu'elles soient étudiées, ni a fortiori comprises.

Nous présenterons alors la formation chirurgicale selon trois aspects, épistémologique institutionnel et épistémique. Puis nous préciserons les notions de contrat et de milieu didactiques, que nous convoquons pour l'étude. Ces notions, issues de la didactique des mathématiques, nous paraissent présenter une part de généricité qui permet leur usage pour l'étude de phénomènes de formation spécifiques des savoirs, mais qui peuvent être autres que mathématiques (Sensevy, 2002 ; Mercier, Schubauer-Leoni, et Sensevy, 2002). Nous développerons ensuite notre approche de l'activité professionnelle, à partir de celles de Vergnaud $(1991,1996)$ et de Balacheff (1995). Nous pourrons alors présenter les outils qui nous permettront l'analyse des interactions didactiques à partir des données recueillies. Cette analyse sera développée dans la quatrième partie, et illustrée d'extraits de protocoles.

\section{ENTRE TECHNOLOGIE ET PÉDAGOGIE : LE VIDE DIDACTIQUE DES TRAVAUX SUR LA FORMATION AU TRAVAIL}

Cette partie présente un aperçu de la littérature portant sur la formation au travail, et en particulier la formation en chirurgie. Elle nous permet de situer notre propre étude par rapport aux travaux existants, dont nous fournissons quelques références pour chacun des courants de recherche identifiés. 
Dans un premier temps, considérons de manière large les travaux qui portent sur la formation au travail, y compris hors du domaine de la santé. Une partie de cette littérature sur la formation par compagnonnage, apprentissage ou transmission filiale relève des domaines historique, sociologique ou ethnographique (Legoux, 1972 ; Delbos et Jorion, 1984 ; Chevallier, 1991 ; Guédez, 1994 ; Ramé et Ramé, 1995). Ces études décrivent les relations entre le maître et l'apprenti, l'environnement de formation, les valeurs transmises et l'évolution du métier. On y trouve quelques éléments précis sur les ficelles du métier, les outils et certaines genèses instrumentales (comme sur le maniement du las en saliculture, Delbos et Jorion, 1984, p. 131). Même s'ils ne traitent pas de l'internat, ces travaux nous fournissent des clés de lecture de son fonctionnement institutionnel, analogue en plusieurs aspects au compagnonnage (Peneff, 1997 ; Vial, 1999 ; Wicart, 2003) : ils ont ainsi un pouvoir explicatif pour notre contexte d'étude. Cependant l'étude de la relation entre le maître et l'élève y est faite sans considérer ce qui les réunit, les occupe et leur pose problème : les savoirs. Cette « réduction pédagogique » des faits de formation, pointée en particulier par Chevallard (1997, p. 46), est une difficulté très présente dans la littérature sur la formation professionnelle. Les travaux portant sur les compétences, issus cette fois des sciences de l'éducation, n'échappent pas, tout au contraire, à cette réduction (Le Boterf, 2010), de même que le courant plus anglo-saxon de l'apprentissage par expérience (experiential learning chez Kolb, 1984, ou informal workplace learning chez Eraut, 2004). Enfin, l'approche plus récente de la didactique professionnelle, qui s'appuie sur les didactiques des disciplines, l'ergonomie cognitive et la psychologie du travail, a plus traité l'identification et la transposition des savoirs professionnels que l'étude de leurs modes de transmission in situ (Pastré, 2008).

La littérature sur la formation initiale et continue des chirurgiens est, quant à elle, dominée par les travaux liés au développement de technologies. En effet, durant ces dernières décennies le développement important de logiciels de guidage et de planification des gestes chirurgicaux, d'abord à vocation professionnelle, a peu à peu amené une réflexion sur les possibilités d'usage de ces outils pour la formation et la certification (Marescaux, Mutter, Soler, Vix, et Leroy, 1999 ; Troccaz, Henry, Laieb, Bosson, et Pichot, 2000 ; Tonetti, 2003). Ce mouvement a été favorisé par une prise de conscience accrue de certaines limites de la formation traditionnelle au bloc opératoire : elle est potentiellement dangereuse pour le patient, peu compatible avec l'augmentation des rythmes de travail et, dans certains pays, on assiste à un refus croissant des patients de prendre part à la formation (Reznick, 2005). En outre la question relativement nouvelle de la formation continue des praticiens entraîne une nécessaire réflexion sur l'évaluation, à laquelle ces outils paraissent pouvoir également contribuer. Ces travaux sont pour la plupart issus de laboratoires d'informatique collaborant avec des services hospitaliers, et adoptent une approche prescriptive. La formation y est principalement considérée dans la dimension d'entraînement au geste, par sa répétition hors du bloc opératoire, rendue possible par de nouvelles techniques de visualisation. Il y est souvent postulé un lien direct entre le niveau de réalisme des dispositifs et la qualité de la formation, et considéré " comme axiome le bienfait et la valeur ajoutée des artefacts technologiques pour le travail et la formation » (Vadcard, de Vries, et Baillé, 2009). Ainsi, ces recherches conduisent à une profusion de réalisations pratiques (simulateurs, logiciels), qui sont parfois associées à des études de satisfaction menées auprès des étudiants. Plus rares sont les travaux qui questionnent ce paradigme dominant ou qui tentent de prendre la mesure des effets de ces dispositifs (Peugnet, Dubois, et Rouland, 1998 ; Nyssen, Larbuisson, Janssens, Pendeville, et Mayné, 2002). Les avancées scientifiques de cette approche se situent principalement au niveau technologique (suivi des apprenants, techniques de visualisation et de reconstruction des volumes anatomiques, etc.). L'objet n'est pas de mener une réflexion sur l'apprentissage, et la dimension formative des interactions entre interne et chirurgien lors des interventions est, de ce fait, peu considérée dans ces travaux.

Nous identifions par ailleurs une littérature traitant de pédagogie médicale, mais qui se préoccupe principalement du premier cycle des études de médecine. Ces travaux questionnent le modèle magistral d'enseignement en vigueur jusqu'à ces dernières années dans la plupart des facultés de médecine, développent des travaux sur l'apprentissage du raisonnement clinique (Barrows et Feltovich, 1987 ; Eva, 2005) et se tournent vers des modèles pédagogiques d'apprentissage par problèmes (Albanese et Mitchell, 1993), de mises en situations cliniques (Barrows, 1987). Malgré l'ampleur des réalisations 
menées au niveau des études de médecine, on note relativement peu d'écho sur la formation pratique des chirurgiens sur le lieu du travail, qui n'a pas à notre connaissance été étudiée dans cette approche.

Finalement, les études portant sur le bloc opératoire restent rares. En psychologie, quelques travaux s'intéressent à la gestion collaborative du travail (McCulloch et al., 2009 ; Stegmann, Zottmann, Siebeck, et Fischer, 2009 ; Blavier et Nyssen, 2010), ou plus particulièrement aux mécanismes d'erreurs (de Keyser et Nyssen, 1993 ; Reason, 1995). Plus rares encore sont les études sur la formation au bloc opératoire. Nous les abordons ci-après.

Lyon souligne comme nous la faiblesse des travaux sur la formation au bloc, de par leur nombre et de par leur approche de l'enseignement et de l'apprentissage, " unidimensionnelle et statique » (2004, p. 1285), conduisant pour la plupart à des études quantitatives cherchant à en mesurer la qualité sans les avoir préalablement étudiés (Hauge, Wanzek, et Godellas, 2001). Pour sa part, elle considère la formation au bloc comme une interaction sociale, et en recherche les facteurs facilitants ou bloquants. Ses travaux peuvent se rapprocher de ceux de Billett (2011), qui identifie également des éléments favorables à la réussite de la formation intentionnelle au travail, comme la mise en place d'un curriculum, et l'explicitation d'une pédagogie (énoncer les objectifs et les rôles, faire participer progressivement, etc.). Mais, si ces travaux amènent une vision plus interactionniste de la formation, ils restent cependant ancrés dans une approche prescriptive qui considère les phénomènes d'enseignement et d'apprentissage de manière détachée des contenus, et se centrent sur une normalisation des techniques de communication pour améliorer la formation. Qui plus est, ces études se basent parfois sur des enquêtes par questionnaires pour identifier les caractéristiques de la situation (Lyon, 2003), alors même que ces mesures auto-rapportées par les étudiants ou les chirurgiens de leur ressenti de la formation ne permettent pas d'en comprendre le fonctionnement. La légitimité des prescriptions faites à la suite de ces mesures est alors, à notre avis, peu fondée.

Citons pour finir le travail d'une linguiste qui étudie les indices, dans le geste et dans le discours du chirurgien, des passages de l'opération routinière à la démonstration à des fins d'enseignement. Ses résultats montrent une dialectique entre règle prescrite générale et conduite particulière, qui va dans le sens de l'identification d'une interdépendance des deux enjeux de travail et de formation: « le passage de la routine à l'énoncé de la règle ne laisse pas le geste intact, mais le reconfigure profondément » (Mondada, 2002, p. 315). Nous reviendrons sur cette question des liens entre le général et le particulier dans la suite du texte.

Finalement, au-delà du constat du nombre réduit d'études portant sur la formation des internes, nous retenons de ce tour d'horizon des recherches sur la formation au travail une focalisation sur les aspects contextuels et organisationnels de la formation, et sur les modes de communication de ses différents acteurs. La pédagogie domine les études sur la formation médicale, et la nature des savoirs en jeu n'est pas questionnée. On note également une forte volonté de prescription, liée au souhait d'améliorer la formation. Cette vision " normalisante et techniciste » (Sarrazy, 1995, p. 85) de la formation conduit par exemple Lyon à préconiser la négociation d'un contrat entre l'étudiant et le chirurgien permettant de définir au préalable les rôles de chacun au cours de l'intervention (2003, p. 687).

Il nous semble au contraire important de sortir de la croyance qu'il existe un ensemble de bonnes pratiques qui pourraient être dictées au maître ou à l'apprenti. Le fonctionnement du système didactique est dépendant de l'enjeu de savoir qu'il porte, et ne peut être ainsi modifié à l'envi (Mercier, 2008). La compréhension des phénomènes qui constituent ce système est une étape primordiale à une quelconque démarche d'ingénierie. Ainsi, nous pensons nécessaire d'enrichir ces travaux par une approche didactique de l'étude de la formation au bloc opératoire, permettant d'en comprendre le fonctionnement. Ce faisant, nous souhaitons dépasser le discours tenu sur l'imitation, l'observation, ou encore l'engagement, en avançant sur leur caractérisation. En nous appuyant sur le cas particulier de l'internat nous espérons, tout en étudiant ses spécificités, être à même d'identifier des phénomènes d'enseignement et d'apprentissage susceptibles de participer à la caractérisation plus générale de la formation sur le lieu du travail.

\section{QUELQUES JALONS ÉPISTÉMOLOGIQUES, INSTITUTIONNELS ET ÉPISTÉMIQUES}

Les travaux didactiques ont montré l'importance d'adopter une perspective systémique pour l'étude des situations de formation. En particulier, 
les contraintes qui pèsent sur l'enseignement et l'apprentissage peuvent être considérés selon trois dimensions, qu'Artigue (1990) qualifie d'épistémologique, de cognitive et de didactique. Les contraintes épistémologiques sont étudiées selon une approche historique et disciplinaire, conduisant à comprendre les développements de la notion à l'étude, ainsi que les filiations et les ruptures qu'elle entretient avec des notions connexes (Vergnaud, 1991). Les contraintes cognitives sont liées au développement du sujet apprenant, et à ses conceptions relatives à l'objet d'enseignement. Les contraintes didactiques sont d'ordre institutionnel, " associées aux caractéristiques du fonctionnement du système d'enseignement $»$ (Artigue, 1990, p. 289).

Nous développons dans cette partie des éléments permettant d'éclairer le lecteur sur ces dimensions.

\section{La chirurgie et sa formation : une histoire marquée par l'empirie et le compagnonnage}

Rappelons ici ce qui a pu devenir une évidence grâce aux travaux de didactiques des disciplines, à savoir que la nature des contenus à transmettre détermine en partie la nature des processus de leur transmission. Dans le cas de la chirurgie, l'histoire nous montre un fort ancrage empirique.

L'internat, notre contexte d'étude, est plutôt récent puisqu'il n'apparaît qu'après la révolution française, lorsque la formation des chirurgiens se joint à celle des médecins. Jusque-là, les modes de construction, de validation, et de transmission des connaissances en chirurgie sont principalement empiriques, conformément à ce que laisse entendre l'étymologie (chir, $\eta \chi \varepsilon 1 \rho$, la main). En Occident, dès le $1^{\text {er }}$ siècle mais de manière encore plus marquée au moyen âge, les médecins se détournent du contact avec le corps pour se consacrer à la théorie, et ce sont les chirurgiens (également barbiers au moyen âge) qui manipulent les instruments pour le soin et la dissection (Sakka, 1998 ; Dachez, 2008). La transmission de leurs pratiques relève de l'apprentissage (formation d'un apprenti au cours de l'activité) et n'est que peu contrôlée par les autorités, tout comme l'exercice même du métier. Quelques précurseurs, dont Vésale $\left(16^{\mathrm{e}}\right)$ et les membres de la confrérie du Collège Saint Côme $\left(13^{\mathrm{e}}\right)$ cherchent à établir une reconnaissance de la profession, et à la différencier de la barberie ignorante. C'est un combat long, dont la victoire est acquise en 1743 (voir ici encore Dachez, 2008, p. 500, ou Rabier, 2010). Quant à l'enseignement, en 1750 l'hôpital ouvre ses portes à la formation des médecins. On reconnaît la nécessité de confronter l'étudiant au réel. C'est le début (ou plutôt la redécouverte, car ces pratiques avaient déjà existé auparavant) de la formation au chevet du malade, instaurée pour développer le discernement des étudiants. Délibérément, le mode de formation pratique des chirurgiens, au contact des patients, est donc en partie adopté pour la formation des médecins, qui souhaite devenir moins professorale. On décide ainsi d'intégrer ces deux modes de formations, que la séparation avait rendus inefficaces, produisant - en grossissant le trait - des barbiers ignorants et des médecins incapables. Mais il s'agit encore seulement de la formation des médecins, et ce n'est paradoxalement qu'avec l'abolition des communautés de métiers en 1791 que la chirurgie est reconnue comme une spécialité de la médecine. La chirurgie accède alors au statut tant souhaité par Vésale dès le début du XVI ${ }^{e}$ siècle, conciliant érudition et empirie. Par suite l'internat est instauré en 1802, répondant à différents objectifs (Vial, 1999) : social - le recrutement par concours remplace la désignation par les maîtres et l'instauration d'un salaire permet un accès aux moins riches; professionnel - les internes constituent les équipes de nuit ; pédagogique - l'apprentissage comprend désormais l'observation des maîtres, les discussions entre pairs, la prise en charge des externes, et un apprentissage de la clinique par la confrontation des hypothèses diagnostiques aux résultats des autopsies (qui étymologiquement désigne l'action de voir de ses propres yeux).

Actuellement la formation au métier de chirurgien est l'une des plus longues qui soient en France. Elle comporte en premier lieu six années d'études de médecine réparties en deux cycles de trois ans, le premier plutôt académique, consacré à l'acquisition du corpus établi des connaissances (anatomie, physiologie, etc.) et le second, l'externat, organisé sous forme d'alternance entre stages et enseignements à la faculté. Viennent ensuite les cinq années d'internat, qui comportent quelques enseignements sous forme de cours ou de séminaires, mais se composent principalement de la participation à la vie du service : réunions, visites quotidiennes des patients, bloc opératoire.

Cette période dans la formation du chirurgien constitue son entrée dans la vie professionnelle. Il y 
apprend les gestes techniques bien sûr, mais également les règles de travail collectif, les routines de préparation, etc. C'est une éducation globale, au sens où l'entend le compagnonnage (Guédez, 1994). Quotidiennement, l'interne se présente ainsi au bloc opératoire en même temps que le chirurgien. Il se lave et s'habille comme lui, ce qui lui donne accès à la zone stérile de la table d'opération et lui permet le contact avec le patient. Il assiste ainsi de très près à l'intervention, et est guidé par le chirurgien qui l'invite à observer, lui fournit parfois des explications sur l'activité qui se déroule, et l'amène peu à peu à prendre part à certaines étapes de l'intervention. L'apprentissage se fonde ainsi sur l'observation, éventuellement commentée et questionnée, et la prise en main progressive du geste. Au terme de son apprentissage, le chirurgien est un professionnel capable de faire son métier, aux deux sens du terme soulignés par Clot, Fernandez, et Scheller (2007) : l'exécuter et le créer, c'est-à-dire participer à son évolution. Lui incombe également une mission de formation : comme chez les Compagnons du Devoir, celui qui sait devra former à son tour (de Castéra, 1996).

Les situations de formation que rencontre l'interne sont ainsi les situations de soins. Elles préexistent à l'intention d'enseigner, et ont des enjeux et des contraintes propres qui peuvent être distincts de ceux de la formation (la contrainte temporelle, par exemple). Le parcours de formation de l'interne, s'il est programmé en lieux et spécialités, par semestres, se constitue ensuite dans la contingence des services et des cas traités pendant la période. Il dépend également de la rencontre avec les " maîtres », les chirurgiens du service, qui n'ont pas tous la même approche de la formation des internes, ni tous les mêmes techniques opératoires. Car si au bloc opératoire un seul chirurgien est présent, l'interne assiste au cours du semestre aux interventions des différents chirurgiens du service. Cette diversité de références est assumée comme une composante importante de la formation, tout comme les deux semestres hors spécialité et les périodes obligatoires de stage dans un hôpital non universitaire, qui servent à compléter les expériences de l'interne. Les rencontres avec d'autres techniques, d'autres modes d'interventions, sont reconnues comme favorisant le progrès et la capacité d'innovation. Comme dans le compagnonnage traditionnel et son Tour de France, on parfait sa propre technique en y intégrant les spécificités d'autres maîtres ou d'autres spécialités.
Cette première partie historique et contextuelle aura montré la nature très empirique de la chirurgie, et dévoilé également une part de l'organisation institutionnelle de l'internat. Cette période de l'apprentissage est présentée comme indispensable par la communauté, et souvent explicitement qualifiée de compagnonnage (Testas, Mouiel, et Delaitre, 1995 ; Vial, 1999 ; Wicart, 2003 ; Becmeur, Grandadam, Kirch, et Mutter, 2004). L'interne doit y constituer sa propre expérience, et le chirurgien est là pour l'y aider, tout en garantissant l'enjeu sanitaire de la situation.

Nous souhaitons comprendre comment le maître accompagne cette prise d'expérience, comment il la guide tout en permettant qu'elle soit bien celle de l'étudiant, qui doit pour cela y engager ses propres connaissances. On retrouve ici une configuration décrite par Brousseau (1998), du jeu de l'élève contrôlé par le maître mais qui doit cependant rester celui de l'élève. Notre hypothèse est que l'enjeu de travail (l'enjeu sanitaire) est partie intégrante de cette situation de formation, et qu'il détermine en partie les relations didactiques qui se mettent en place autour de l'enjeu de formation.

Le paragraphe suivant apporte des éléments sur la dimension épistémique de l'étude en précisant la nature des savoirs en jeu dans cette situation.

\section{Nature des savoirs en jeu : présentation de l'objet de l'étude}

Pour pouvoir mener cette étude didactique, une réduction épistémique est indispensable : nous ne traiterons qu'une classe de situations de la chirurgie orthopédique, celle du vissage ilio-sacré, dont le nom traduit bien le fait de placer une vis dans la zone de l'articulation entre l'aile iliaque et le sacrum, dans le cas de lésions de l'anneau pelvien. Nous avons développé ailleurs (Vadcard, Tonetti, et Dubois, 2011) une analyse détaillée de cette activité, dont nous reprenons ici quelques points essentiels.

L'enjeu sanitaire de l'activité peut-être énoncé comme celui de prodiguer le meilleur soin possible en minimisant la fragilisation du patient (temps d'anesthésie, irradiation, plaies et hématomes iatrogènes, etc.). Cette activité n'est pas isolée, elle s'insère dans un environnement plus large dont font partie les anesthésistes, les manipulateurs radio, les instrumentistes, etc. Sans minimiser l'importance 
de la dimension collective du travail, nous nous permettons ici d'isoler l'activité du chirurgien car, si elle dépend de cet environnement, elle le guide également, étant centrale dans l'objectif partagé par tous. En outre, une étude plus poussée au regard des contenus requiert une focalisation qui n'est pas un déni du reste, mais un nécessaire isolement pour l'analyse de phénomènes qui pourront ensuite être replacés dans un contexte plus large.

La vis (ou les deux vis dans certains cas) est implantée pour maintenir en position de reconstruction les éléments impliqués lors d'une lésion de la zone. Deux types de lésion peuvent se rencontrer, les disjonctions et les fractures, qui définissent deux sous-classes de situations. Dans le cas d'une disjonction de l'articulation, le rôle de la vis sera d'exercer une compression entre l'aile iliaque et le sacrum pour qu'elle puisse se reconstituer. Dans le cas d'une fracture du sacrum, la vis est placée de manière à maintenir sans compression les parties osseuses, et permettre la reconstruction naturelle de l'os. Les fractures, comme les disjonctions, sont classées selon leur nature et leur gravité (Tonetti et al., 2000). Pour ce qui nous occupe ici, nous n'avons pas besoin d'entrer dans ces nuances.

Figure 1 - Anatomie du bassin

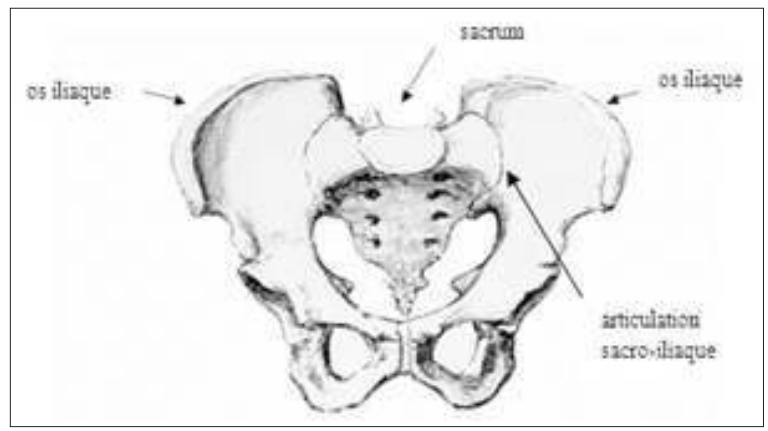

La vis est placée de manière percutanée, c'est-àdire sans plus d'incision cutanée que celle qui permet son passage. Ces techniques percutanées, largement développées dans plusieurs domaines chirurgicaux, permettent d'obtenir une diminution importante de la morbidité et du temps de récupération postopératoire du patient. On en perçoit donc facilement l'enjeu sanitaire. Nous en verrons plus loin certaines conséquences sur la formation. Le geste opératoire percutané en chirurgie orthopédique est effectué sous le guidage de radios qui sont prises régulièrement au cours de l'intervention, et fournissent des indications au chirurgien sur la mise en place de sa vis.
Figure 2 - Contexte opératoire de la chirurgie orthopédique percutanée (le patient est allongé, le chirurgien manipule l'appareil de radioscopie et l'écran permet la visualisation des radios prises)

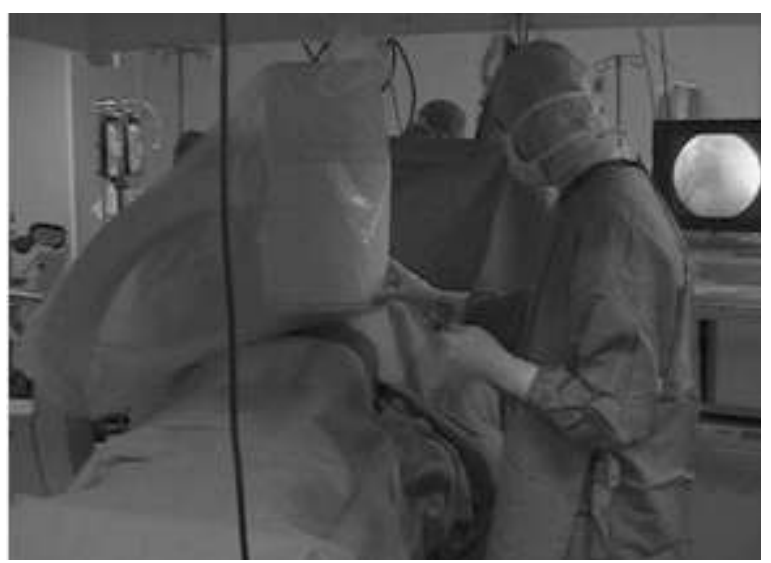

Plusieurs étapes sont nécessaires au bon positionnement de cette vis. Tout d'abord, le chirurgien repère certains éléments de la structure osseuse du patient : il positionne une barre métallique sur le corps du patient à l'endroit où il considère qu'est placée la structure qu'il veut repérer (la partie supérieure du sacrum, ou le prolongement du fémur, par exemple); il prend une radio pour vérifier la superposition de ces deux éléments (l'os et la barre); il modifie la position de la barre et prend des radios jusqu'à ce que la superposition soit bonne; enfin il trace le long de la barre sur la peau du patient un trait qui lui donnera tout au long de l'intervention un repère visuel des structures osseuses.

Figure 3 - Le repérage cutané

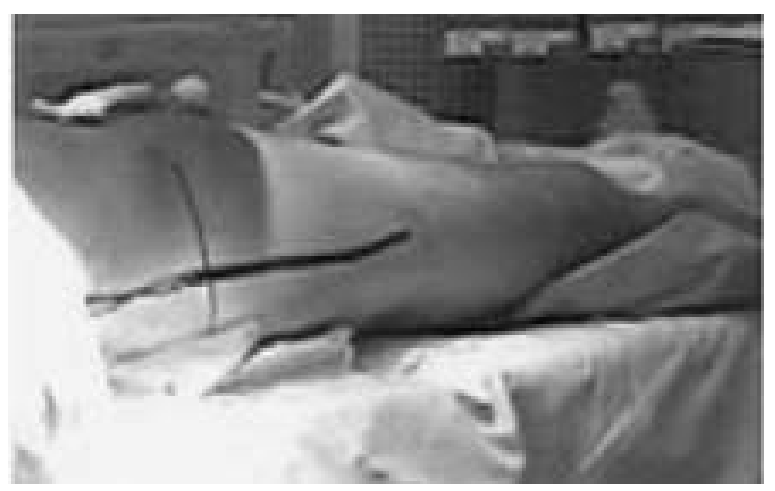

Il insère ensuite une broche (tige métallique pointue en l'une de ses extrémités) à travers la peau, puis à travers l'os. Le trajet de la broche détermine celui de la vis canulée qui sera insérée le long 
de la broche. Ce trajet doit permettre de donner à l'ensemble vis et pièces osseuses une partie des propriétés mécaniques qui servent l'enjeu opératoire, à savoir permettre une bonne reconstruction osseuse naturelle. Dans le cas d'une fracture, la vis doit être perpendiculaire à la lésion, et elle doit s'avancer jusqu'à plus de la moitié du corps vertébral de la dernière vertèbre sacrée (S1) pour avoir un bon ancrage, c'est-à-dire résister aux forces inverses qui tendent à l'arracher du sacrum. Dans le cas d'une disjonction, la vis doit être perpendiculaire à l'articulation, et peut être moins avancée dans le corps de S1. En effet, l'ancrage est en partie fourni par le filetage de la vis qui doit être positionné de part et d'autre de l'articulation. Dans le cas de la fracture, le maintien sans compression implique de ne pas positionner le filetage à l'endroit de la fracture, l'ancrage doit alors être obtenu au niveau de l'extrémité de la vis. En outre, le trajet de la broche doit impérativement
« outlet »), de manières isolée ou conjointe, qui lui permettent de se représenter la position de sa broche dans la structure osseuse du patient. Cette caractéristique de l'activité est une contrainte forte du point de vue de la formation. D'une part elle implique, pour pouvoir agir, des traitements complexes pour obtenir une représentation efficace de la position de la broche dans le volume osseux (voir encore pour cet aspect, Vadcard et al., 2011). D'autre part elle modifie considérablement les possibilités d'observation de l'activité pour l'interne. L'observation est en quelque sorte conditionnée par l'élaboration d'une représentation de la zone d'activité opératoire, représentation qui fait justement partie des enjeux de la formation.

Enfin, lorsque la broche est positionnée de manière satisfaisante, une suite de manipulations d'instruments canulés permet de tarauder l'os puis d'y introduire la vis. La broche est alors ôtée, et l'incision cutanée refermée par un point de suture.

Figure 4 - Positionnement de la vis à partir de celui de la broche

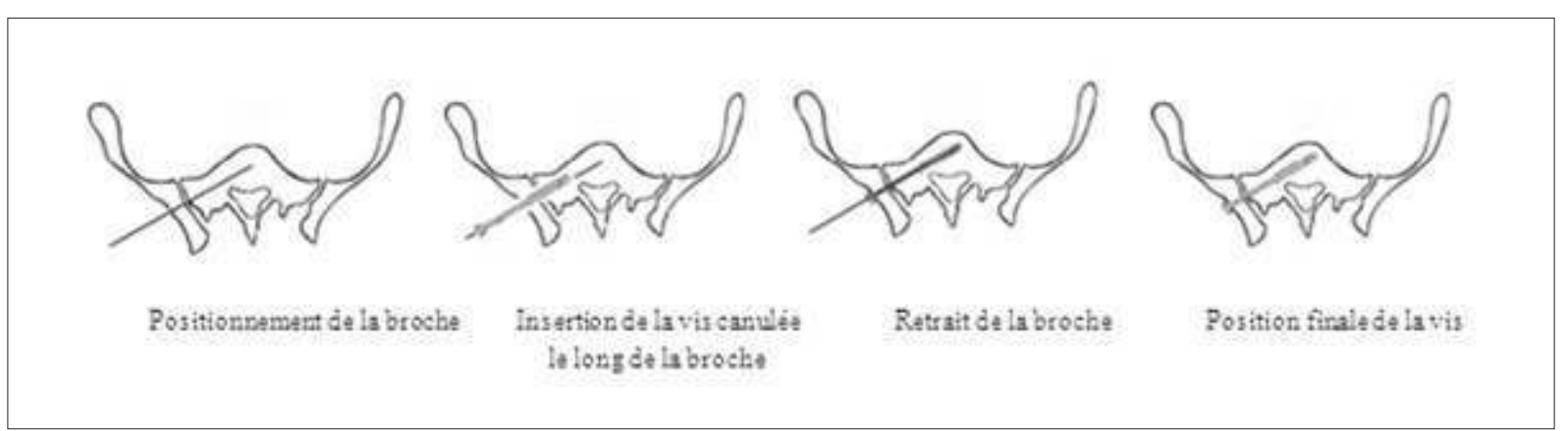

définir une position strictement intra-osseuse à la vis. Or la structure osseuse concernée comporte des zones étroites, par lesquelles la vis devra passer sans sortir. Le risque de trajet extra-osseux est important, que ce soit du fait du trajet initial de la broche ou de l'épaisseur de la vis par rapport à celle de la broche, qui entraîne un risque lorsque la broche passe près $\mathrm{du}$ bord de l'os. L'environnement nerveux de cette zone est très sensible, il faut en éviter les lésions qui peuvent avoir de graves conséquences pour le patient.

L'insertion de la broche est, on l'aura compris, la phase la plus importante de l'intervention. Elle porte une grande part des enjeux et des contraintes de la situation. Lors de cette étape, le chirurgien prend de nombreuses radios qui lui permettent de suivre la progression de sa broche. Plusieurs incidences sont utilisées (nommées « face », « profil », « inlet » et
La procédure de l'intervention, décrite en termes d'actions, est ainsi assez simple. Au tout début d'une des interventions observées le chirurgien la résume ainsi à l'interne : «Donc, tu prends ton machin [ = la broche] comme ça, tu perces la peau tu te mets au contact de l'os et tu donnes un petit coup de marteau. Ensuite tu fais tes inlet/outlet, inlet/outlet [ = radios], tant que c'est bon tu pousses. Avant de pousser plus loin tu fais ton profil [ = radio] ». Mais le lecteur aura bien compris que des difficultés importantes sont à chercher dans le caractère percutané de l'acte. L'accès indirect à des informations visuelles sur l'action est une contrainte importante de la situation. Qui plus est, ces informations n'ont de sens que pour celui qui sait les déchiffrer. Ainsi par exemple, à mi-course le chirurgien prend des repères sur deux incidences radios (inlet et outlet qui fournissent des projec- 
tions selon deux directions perpendiculaires, et sont utilisées conjointement) pour valider sa trajectoire, et couple ces informations visuelles avec le ressenti d'une zone de faible résistance précédée de deux passages, rapprochés, de plus grande résistance. L'ensemble lui fournit une indication précise de l'emplacement de sa broche dans l'os, à condition toutefois que les radios prises vérifient elles aussi des critères de validité intrinsèques qui en font de «bonnes » radios, c'est-à-dire montrant bien la projection qu'elles sont censées montrer. Le critère extrinsèque qu'est l'angle du rayon par rapport à la table ne suffit pas, le patient pouvant être « tordu », plus ou moins cambré, ou simplement non strictement aligné avec le bord de la table.

L'activité est ainsi constituée d'une suite d'actions, d'apparence peu complexes, mais qui nécessitent de continuelles activités de contrôle, menées par des prises d'informations, formelles ou informelles au sens de de Montmollin (1974, p. 35), qui s'organisent en systèmes (ibid., p. 37). Nous identifions suite à l'analyse de l'activité que l'enjeu majeur de la formation réside dans cette dimension de contrôle et son couplage avec les actions menées. La procédure opératoire ne présente en effet aucune difficulté de compréhension pour qui connaît l'anatomie de la zone concernée, mais ne suffit pas au déroulement de l'activité (Pastré, 2001). On ne saurait donc étudier cette activité, et sa formation, en se contentant du niveau initial d'analyse que fournit la procédure prescrite qui n'épuise pas, loin de là, la compréhension de «l'intelligence de la tâche » (de Montmollin, 1984). C'est pourquoi nous présentons ci-après les possibilités que nous nous donnons d'étudier plus finement l'activité de travail et de formation au bloc opératoire.

\section{NOTIONS CONVOQUÉES POUR L'ÉTUDE}

Rappelons avant la suite de l'étude que les situations de formation qui nous occupent fonctionnent bien, et depuis fort longtemps. L'objet n'est ainsi pas de rechercher un éventuel dysfonctionnement, mais bien d'en obtenir une description armée par nos objectifs de recherche, qui permette de les caractériser, et d'en comprendre le fonctionnement. Car au premier abord il peut sembler que l'« artificialité constitutive des actes didactiques » (Johsua, 1997, p. 23) ne soit pas aisée à instaurer au bloc opératoire. L'une des conclusions de ce texte sera que cette arti- ficialité se situe sur le plan du contrat (Brousseau, 1998), dans les relations qui se nouent entre maître et apprenti au cours de la situation.

\section{Coutume, contrat et milieu didactiques}

Le contrat didactique est défini comme « une relation qui détermine - explicitement pour une petite part, mais surtout implicitement - ce que chaque partenaire, l'enseignant et l'enseigné, a la responsabilité de gérer»(Brousseau, 1998, p. 61). La relation didactique étant dynamique, c'est, précise Brousseau, moins le contrat lui-même mais «le processus de recherche d'un contrat » (ibid., p. 62) qui permet de la modéliser et de l'expliquer, notamment en termes de jeux, celui du maître et celui de l'élève, qui se déroulent en classe. Balacheff (1988) propose d'adjoindre à la notion de contrat celle de coutume. Ce faisant, il souhaite considérer deux temporalités. L'une, plutôt longue, correspond à la construction des habitudes de fonctionnement du sous-groupe considéré, au sein de l'institution didactique. C'est le niveau de la coutume, qui se construit sur l'année scolaire pour une classe. L'autre temporalité, plus brève, est liée à une situation d'enseignement particulière. On retrouve ici le contrat tel que défini par Brousseau, mais qui est alors vu comme étant subordonné à la coutume : " la coutume pèse sur la négociation du contrat didactique, notamment en délimitant ce qui est négociable de ce qui ne l'est pas » (Balacheff, 1988, p. 22). La coutume fournit un cadre à la mise en place de contrats plus locaux, relatifs à une situation de formation particulière. Et bien que de nature plus institutionnelle que le contrat, elle reste spécifique du savoir enseigné.

Il nous semble que la considération conjointe de ces deux notions présente un intérêt pour l'étude de la formation en situation de travail, en ce qu'elles permettent d'identifier et d'expliquer des phénomènes dont certains présentent un caractère de stabilité, et d'autres sont plus variés, donnant à voir la nature dynamique de la relation didactique. Nous pensons par exemple à un phénomène similaire à celui que Brousseau nomme «le paradoxe de la dévolution des situations » (Brousseau, 1998, p. 73) : l'interne doit résoudre le problème posé, mais s'il le résout sans faire lui-même les choix, les raisonnements nécessaires, le fait d'avoir résolu le problème n'est pas l'indice d'un apprentissage, au sens de la 
capacité de le «mettre en ouvre de lui-même dans des situations qu'il rencontrera en dehors de tout contexte d'enseignement » (ibid., p. 59). Mais de son côté le chirurgien est contraint, du fait de sa responsabilité sanitaire vis-à-vis de la situation, de fournir à l'interne les éléments de sa résolution. D'autant plus que la solution émerge de l'activité, finalisée et sous contraintes : elle n'est pas connue d'avance. Le chirurgien est partie prenante de la recherche de la solution, en même temps qu'il enseigne à l'interne cette démarche, qui permet de déterminer une solution parmi l'ensemble des possibles, qui respecte au mieux les enjeux et les contraintes du problème. Le maître dénature ainsi en partie la relation de l'apprenti à la situation, mais autorise du même coup son existence qui, sans cela, ne serait pas possible: il permet à l'interne de s'engager dans la situation, de la prendre en charge, tout en sachant que celuici n'a pas les moyens de la mener à son terme de manière autonome. Le maître régule alors la relation de l'interne à la situation, modifiant les termes du contrat au fur et à mesure que la situation évolue, de manière à maintenir cette relation tout en en préservant l'issue, qui se doit d'être favorable dans un temps relativement contraint : il mène conjointement la réalisation des deux enjeux qu'il poursuit.

Comment, dans ces conditions, l'apprentissage peut-il avoir lieu ? Nos protocoles vont nous permettre de décrire certains aspects de la coutume partagée par le maître et l'apprenti. Ce dernier va chercher à reproduire, à reconstruire, les règles et heuristiques qui lui sont partiellement dévoilées, de manière à remplir son devoir d'apprenti. Le maître, lui, joue le jeu de l'autonomie de l'apprenti face à la situation, tout artificiel que ce jeu puisse paraître à certains moments. Ce leurre de responsabilité permet et facilite le jeu de l'apprenti. Il se rapproche de ce que Delbos et Jorion notent à propos des saliculteurs : il faut « s'y voir » $(1984$, p. 144), c'est-à-dire avoir une image de soi « en position anticipée de maîtrise » (ibid.). Et « s'y voir » dans un domaine empirique comme celui de la chirurgie, cela nécessite de toucher, de prendre part au geste.

Coutume et contrat permettent au maître d'instaurer les conditions de l'apprentissage, en déterminant en particulier la nature du milieu. Ce dernier est ce qui résiste à l'élève, lui permettant de percevoir l'insuffisance de ses interventions, et de trouver les moyens de les ajuster. Il est constitué d'éléments matériels de la situation, mais également en partie des réactions des autres élèves (en classe), et des interventions du maître. Nous verrons que dans le cas qui nous occupe, les interventions du maître constituent la part la plus importante des rétroactions du milieu. L'enjeu sanitaire empêche en partie de laisser le milieu matériel réagir aux actions de l'interne. Le chirurgien sera ainsi l'intermédiaire de ces réactions, en les exprimant sans les laisser se produire. On perçoit ici l'importance de la coutume, précédemment décrite, qui rend viable la relation didactique - qui rend donc possible l'apprentissage malgré la médiation très présente du chirurgien entre l'interne et le milieu matériel.

Enfin, la situation de formation visant par nature sa propre inutilité, le sous-système fondamental dans la théorie des situations didactiques est celui de milieu adidactique, avec lequel l'élève interagit au cours d'une situation dénuée, à ses yeux, d'intention didactique. Tout se passe là comme si le sujet apprenant prenait en charge le traitement de la situation, mettant entre parenthèses la conscience qu'il a de son rôle d'élève, des intentions du maître et de l'institution dans laquelle ils sont engagés. L'internat regroupe en une unité d'espace et de temps les deux types de situations : celle de travail, dont on vise la maîtrise chez l'apprenti, et celle de formation, support à la construction de cette maîtrise. Pour autant, ces situations ont des temporalités distinctes, mais qui se superposent le temps d'une intervention. Le milieu adidactique va alors se déterminer peu à peu au cours de la situation, par les indications que le maître fournit à l'élève : il lui désigne en quelque sorte les éléments qui feront partie de ce milieu avec lequel il devra interagir.

Nous avons présenté dans ce paragraphe les notions de contrat, de coutume et de milieu didactiques, qui serviront notre étude. La section de présentation des résultats permettra de montrer plus en détails certains des phénomènes dont on a esquissé ci-dessus la description. Auparavant, nous présentons notre approche de l'activité professionnelle, toujours dans l'objectif de nous permettre de mieux comprendre les phénomènes observés lors de l'étude des données.

\section{Nature de l'activité professionnelle}

Le métier de chirurgien est classiquement considéré comme associant une dimension gestuelle à une 
part importante de prises de décisions et d'analyse de la situation. Cette dualité n'est pas questionnée dans le cas de la chirurgie, mais l'est plus pour d'autres métiers manuels pour lesquels la part cognitive semble moins évidente. C'est cet oubli du cognitif qui amène de Montmollin (1984) à nous rappeler que le geste technique n'est pas constitué, loin s'en faut, uniquement d'action.

Mais cette dualité, chère également au Compagnon qui aspire à l'union de la main et de la pensée, n'est que peu traitée dans la littérature : dans les travaux sur la formation au travail l'analyse cognitive reste sommaire, se résumant le plus souvent à l'évocation d'une distinction entre théorie et pratique. On pourrait à ce propos reprendre les propositions de Latour et révoquer cette catégorisation, arguant que la théorie résultant d'une pratique, elle ne « désigne aucunement un procès mais seulement un produit » (1996, p. 133). La chirurgie semble particulièrement appropriée à l'examen de cette position. Les quelques éléments historiques que nous avons exposés plus haut pourraient à cet effet être complétés par une étude de la nature et du fonctionnement des aphorismes d'Hippocrate (Prioreschi et Babin, 1995) ou des traités anatomiques de Vésale (Sakka, 1998) dans la formation et dans l'exercice de la profession.

Pour l'étude présente, et afin de traiter la question toujours difficile des rapports entre comportements et cognition, nous proposons, comme le suggère Vergnaud, d'effectuer une « descente vers le cognitif » (2001, p. 8). Cet auteur prône la prise en compte et la compréhension du fonctionnement des invariants opératoires dans l'étude de l'activité humaine (Vergnaud, 1990). Sur ces invariants opératoires repose la production des règles d'action et des anticipations. Celles-ci permettant à leur tour de générer les conduites, qui constituent l'activité du sujet. Les invariants opératoires sont la clé de l'adaptativité de la conduite du sujet, en tant qu'entités sur lesquelles repose l'analyse de la situation : ils "permettent de sélectionner et interpréter l'information pertinente et de la traiter. Le schème qui s'adresse à une classe de situations bien identifiée comporte tous les invariants opératoires nécessaires à la reconnaissance des objets présents dans ces situations et des propriétés et relations qui sont nécessaires à l'évocation des règles d'actions pertinentes, compte tenu du but » (Vergnaud, 1996, p. 285).

Les « règles d'action, de prise d'information et de contrôle » constituent « la partie proprement générative du schème, celle qui engendre au fur et à mesure le décours temporel de l'activité » (Vergnaud et Récopé, 2000, pp. 46-47). Les auteurs précisent que « les règles n'engendrent pas que l'action, mais toute l'activité ; aussi bien les prises d'information et les contrôles que les actions matérielles ellesmêmes » (ibid., p. 47). Cette activité de contrôle est également mise en avant par Balacheff (1995), qui propose de distinguer au sein des règles d'action, celles qui sont engagées vers l'action sur le réel (les opérateurs), de celles qui servent le contrôle de cette action (les contrôles). Nous reprenons cette distinction dans notre étude, et la complétons par la dimension des prises d'information, qui n'est pas explicitée dans ce modèle. Or c'est une dimension particulièrement importante de l'activité professionnelle. Vergnaud nomme « compétence critique » le fait de disposer pour agir de «toute une série d'indices » et de «catégories fines» (1996, p. 279). Ombredane distingue signes primaires et secondaires, selon qu'ils préexistent dans le travail ou qu'ils sont « artificiellement introduit[s] dans le circuit de travail * (1992, p. 15). La même distinction se retrouve chez de Montmollin sous les termes de signes formels et informels (1974, p. 35). Ce dernier propose également de considérer la dimension systémique des signaux du travail, organisés en systèmes simples, redondants ou complexes.

Un objectif de la formation est la construction progressive de ces structures de détection et de traitement de l'information, qui permettent à terme la production d'une activité efficace et adaptée. En effet, si certains éléments de la situation sont des signes pour le chirurgien, ils ne le sont pas d'emblée pour l'interne, qui doit justement élaborer ce qui les rendra signes, c'est-à-dire opératoires pour l'activité. Les interventions du maître, en tant qu'éléments du milieu didactique, porteurs de rétroactions pour l'élève, sont primordiales dans ce processus de construction. Et ce dernier engage une importante dialectique entre le général et le singulier, qui nous intéresse particulièrement dans le cadre de la formation professionnelle car il s'agit bien d'amener l'interne à construire, à partir des situations singulières rencontrées, une capacité à agir qui les dépasse.

Ainsi, un dernier aspect important de l'activité que nous souhaitons considérer ici est la validation, au cours de laquelle est convoquée la structure de contrôle. Au sein de la validation on peut distinguer, comme le proposent Balacheff (1987) et Margolinas 
(1993), les processus de vérification et les processus de preuve. Les premiers sont liés à l'existence d'un doute sur les conséquences d'une action. Ils ont un caractère local, et ne sont pas nécessairement accompagnés d'une formulation. Les seconds ne sont pas liés au doute, mais à la recherche des raisons de la nécessité du résultat de l'action. Ils ont ainsi un caractère de généralité que n'ont pas les processus de vérification.

Le caractère empirique de l'activité chirurgicale nous amène à envisager la présence des deux types de processus : les vérifications (contingentes, ad hoc) sont indispensables à la conduite de l'activité singulière, et apparaissent au sein d'un processus de preuve, plus général. Ce double processus de validation n'est pas sans rappeler le phénomène décrit par Vergnaud (1991) de variabilité des conduites - conduisant à la nécessité de mener des vérifications - au sein de l'invariance de l'organisation de la conduite - validée par un processus de preuve. Les processus de validation, qu'ils relèvent de la preuve ou de la vérification, aboutissent à l'élaboration chez le sujet d'un jugement. Celui-ci n'est pas nécessairement formulé, mais il est indispensable à la poursuite de l'activité : « décision, action, vérification s'enchaînent et interagissent pour chaque temps opératoire » (Tonetti, 2003, p. 121).

Pour conclure cette partie nous illustrons par un bref exemple les notions précédemment exposées :

Le chirurgien possède un ensemble d'invariants opératoires qui lui permettent, par une activité syncrétique (Cornu, 1988) et incorporée (Leplat, 1995), de guider la détection des informations, de les traiter, et de produire des jugements sur l'action menée (« elle [la broche] est bien, là »). Il sait, par exemple, que l'os offre une résistance plus élevée que la peau et la graisse qui le précèdent (information haptique); que les trous du sacrum sont repérables sur une radio outlet (information visuelle) et que l'arrivée de la broche dans la zone des trous est précédée du passage d'une corticale qui procure une sensation de résistance plus forte (information haptique) couplée à un bruit plus aigu du moteur chirurgical (information auditive), si la broche utilisée n'est pas filetée car « avec la broche filetée tu sens pas ce que tu fais alors qu'avec la tige non filetée quand tu butes contre une corticale crrrr, tu vois t'as une sensation alors que là ça te tire vers l'avant t'as pas de sensation » (domaine de validité de l'invariant opératoire permettant de traiter l'information haptique), etc. Un ensemble important de ces invariants opératoires a été identifié lors de l'étude déjà citée (Vadcard et al., 2011).

À ce point du texte, nous pouvons présenter les outils d'analyse, construits à partir des éléments qui précèdent, que nous utilisons pour le traitement de nos données.

\section{PRÉSENTATION DE L'ÉTUDE}

\section{Outils d'analyse}

Afin de caractériser la nature des relations que le chirurgien met en place au bloc opératoire, et d'appréhender les rôles respectifs et conjoints des enjeux de travail et de formation dans la situation, nous nous dotons d'un outil d'analyse des interactions entre l'interne, le chirurgien, et la situation, celle-ci étant constituée des éléments sur lesquels l'interne et le chirurgien agissent; en premier lieu, le patient. Lorsque nous parlons ici d'enjeu de travail, nous effectuons une réduction destinée à faciliter le discours. En effet, comme précisé plus haut, le travail du chirurgien consiste également à former l'interne.

Nous proposons un outil de description des interactions possibles en situation qui prend en compte ces particularités, et qui précise ainsi les interactions « sujet-milieu » classiquement prises en compte en didactique. Considérant trois éléments, l'interne (I), le chirurgien ( $\mathrm{Ch}$ ) et la situation (S), nous définissons cinq actions, codées de 1 à 5 : de l'interne vers le chirurgien (1) ou la situation (2), du chirurgien vers l'interne (3) ou la situation (4), et de l'interne vers la situation, médiée par le chirurgien (5). La dimension gestuelle de l'activité nous amène en effet à considérer la possibilité de guidages physiques de l'apprenti par le maître (Laurençon, par exemple, le note en atelier : « leur prendre la main [...] pour faire le geste avec eux leur faire sentir le travail », 1988, p. 125 ; Billett, 2011, le relève dans des travaux menés sur la poterie). Les actions (flèches dirigées vers la droite sur la figure ci-dessous) sont définies comme des verbalisations ou des gestes destinés à modifier la situation. Les rétroactions correspondantes, codées de a à e, sont les modifications de la situation provoquées par les actions précédentes, et discernables par le sujet. Les couples action-rétroaction définissent des interactions. Précisons que nous ne développons pas à proprement parler d'étude de la communication non verbale. Nous prenons seule- 
ment en compte les gestes déictiques, par exemple ceux qui sont orientés vers le patient ou vers les radios. Enfin, nous considérons classiquement pour l'étude des verbalisations quatre types de phrases: déclaratif, injonctif, interrogatif, exclamatif.

Nous menons ci-après une description a priori de chacune de ces interactions.

Figure 5 - Interactions

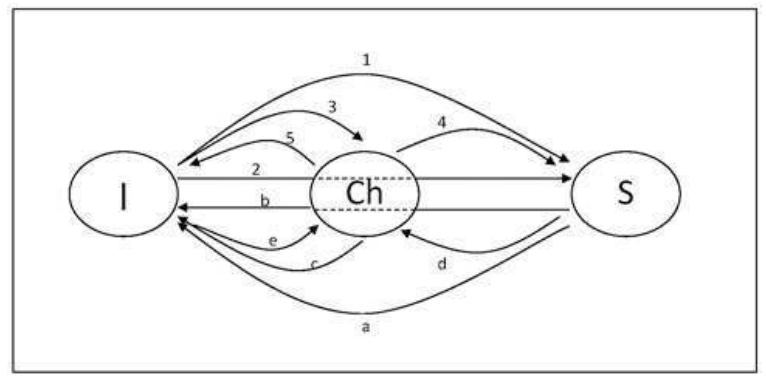

Interaction 1-a : l'autonomie

Le sujet apprenant intervient directement sur la situation, et en détecte seul les rétroactions. Cette interaction peut servir l'enjeu de formation ou l'enjeu de travail selon que l'autonomie de l'interne envers la situation relève ou non d'un choix didactique du chirurgien.

Interaction 2-b : l'activité médiée.

L'interne agit et perçoit les effets de son action par l'intermédiaire (verbal ou gestuel) du chirurgien. Nous identifions cette interaction comme servant l'enjeu de formation.

Interactions 3-c : le questionnement.

Selon le type de phrase initiant cette interaction, ce questionnement peut être :

- 3 déclaratif : un questionnement indirect ;

- 3 interrogatif : un questionnement direct ;

- 3 injonctif : non caractérisé car il ne devrait pas apparaître, étant incompatible avec les positions institutionnelles relatives des acteurs.

Le questionnement à l'initiative de l'interne vers le chirurgien est relatif à l'enjeu de formation.

\section{Interaction 4-d : l'observation}

Le chirurgien est dans une interaction directe avec la situation, ce qui, du point de vue de l'interne, constitue une situation d'observation. Cette interaction relève principalement de l'enjeu de travail, même si elle sert celui de formation. L'action du chirurgien peut entraîner des verbalisations nécessaires à la poursuite de l'action (lorsqu'il fait régler l'appareil de radioscopie, par exemple). Dans ce cas, nous codons 4-d l'interaction. En revanche, si l'action entraîne une verbalisation non indispensable à l'action, nous considérons alors que cette verbalisation est destinée à l'apprenant, et nous codons alors la rétroaction correspondante $b$ (du chirurgien vers l'interne) et non pas d (du chirurgien vers la situation). Les enjeux de travail et de formation sont dans ce cas plus équilibrés.

Interaction 5-e, selon le type de phrase qui l'initie est :

- 5 déclaratif : une explication ;

- 5 interrogatif : un questionnement ;

- 5 injonctif : un ordre.

Ces interactions 5-e servent d'abord la formation, mais dans un équilibre avec l'enjeu de travail qui pourra varier en fonction de leurs contenus.

Nous identifions également des interactions combinant différemment les cinq actions et les cinq rétroactions décrites. Nous avons déjà évoqué l'interaction 4-b, l'observation commentée. Nous identifions également une action de type 5-1, l'action guidée : une injonction du maître provoque l'action de l'apprenti sur la situation. Enfin, signalons l'interaction 1-b, au cours de laquelle le chirurgien commente les résultats de l'action menée par l'interne : l'action autonome commentée.

Au-delà de l'identification des types d'interaction, l'étude envisagée se doit de porter un regard sur la nature des savoirs engagés dans ces interactions. Ainsi nous complèterons l'étude de nos protocoles par une catégorisation des contenus, issue de l'approche de l'activité présentée plus haut, et qui distingue les verbalisations ayant trait à l'action, au contrôle, à la prise d'information, ou au jugement.

\section{Données et démarche}

Notre étude se fonde sur les enregistrements audio et vidéo de cinq interventions de poses de vis ilio-sacrées qui se sont déroulées au CHU de Grenoble entre 2003 et 2010. Chaque intervention rassemble un chirurgien et un interne autour d'un patient. Nous avons filmé deux chirurgiens et quatre internes, selon quatre configurations différentes (deux interventions comportent les mêmes sujets) : 
Tableau 1 - Répartition des sujets et durée des interventions

\begin{tabular}{|l|l|l|l|l|}
\hline & Interne 1 & Interne 2 & Interne 3 & Interne 4 \\
\hline Chirurgien 1 & VSI-1 $(43 \mathrm{mn})$ & VSI-2 $(12 \mathrm{mn})$ & VSI-5 (13 mn) & \\
\hline Chirurgien 2 & & & & $\begin{array}{l}\text { VSI-3 (24 mn) } \\
\text { VSI-4 (30 mn) }\end{array}$ \\
\hline
\end{tabular}

La durée moyenne des interventions est de 24 minutes, avec un écart-type de 11 minutes. Les acteurs sont le chirurgien (Ch), l'interne (I), et le manipulateur de l'appareil de radioscopie (MR).

La caméra est tenue par le chercheur, situé à 2 mètres environ du patient, hors de la zone stérile. La focalisation est faite sur la zone opératoire et sur le moniteur d'affichage des radios.

Ce corpus avait initialement été constitué dans l'objectif d'une modélisation des connaissances liées à cette intervention (Vadcard et Luengo, 2005). De ce fait, certaines caractéristiques des données ne sont pas adaptées au mieux à la présente étude. En premier lieu, ces données ne constituent pas un échantillon, mais seulement un extrait des interventions qui se sont déroulées au CHU au cours de cette période. Ensuite, nous manquons de données sur les internes, puisque ceux-ci n'étaient pas l'objet principal du recueil : le semestre d'internat, la familiarité avec la chirurgie orthopédique percutanée et avec l'intervention de pose de vis ilio-sacrées, les antécédents de travail avec le chirurgien, auraient pu être des éléments de mesure pour qualifier le niveau de l'interne. Pour ces raisons, nos données ne constituent pas un échantillon représentatif : elles ont une valeur heuristique et non probatoire, nous permettant d'éprouver le cadre et les outils d'analyse que nous proposons. Les résultats avancés dans la suite du texte sont à considérer en tenant compte de ces précisions.

Notre démarche d'analyse comporte quatre temps :

Dans un premier temps, l'intégralité des paroles est retranscrite. Sont également notées, placées entre crochets dans les protocoles, les prises de radios et les manipulations de l'appareil de radioscopie, des instruments chirurgicaux et du patient. Certaines précisions et des commentaires sont ajoutés par le chercheur, et figurent également entre crochets (descriptions d'attitudes, précisions sur la référence de certains pronoms, explications sur la situation, etc.). Nous homogénéisons le début et la fin de nos protocoles par des événements repérables : insertion cutanée de la première broche et fin du positionnement de la broche. Cette réduction des données à une période commune à tous les films est indispensable et ne dessert pas l'étude de la formation en cours d'action, même s'il est bien entendu que la relation didactique ne commence ni ne s'arrête avec la manipulation de la broche. Du point de vue chirurgical, nous avions identifié que l'essentiel de l'activité se situait dans le positionnement de la broche. L'installation du patient et le repérage cutané (éléments préalables), le taraudage et le vissage (éléments postérieurs) ne sont donc pas étudiés.

Dans un deuxième temps nous identifions des épisodes, qui sont des ensembles de verbalisations définis en fonction de leur sens au sein de l'activité. Certains épisodes se retrouvent dans tous les films étudiés (insertion cutanée, contrôle de face, fixation, etc.), d'autres sont caractéristiques de l'histoire singulière qui se déroule (manipulation du patient, panne de l'appareil de radios, etc.). Nous obtenons environ 300 épisodes sur l'ensemble des cinq films.

Dans un troisième temps les verbalisations de chaque épisode sont codées en fonction de trois dimensions :

- type d'interaction : les actions sont codées de 1 à 5, les rétroactions sont codées de a à e

- type de phrase : injonction, déclaration, interrogation ou exclamation

- type de contenu : action, critère de contrôle, prise d'information ou jugement.

Dans un quatrième temps les épisodes codés sont étudiés de manières isolée, longitudinale, et transversale :

- Leétude isolée consiste à étudier chaque épisode de manière autonome, pour caractériser les interactions et les contenus qui le constituent. On y identifie par exemple que l'insertion cutanée de la première broche dans le protocole VSI-2 est une action médiée, qui porte sur une action (« insérer ») et un critère de contrôle (« jusqu’à l'os »). 
- Létude longitudinale consiste à examiner la suite des épisodes d'un même protocole, pour obtenir une vision de l'histoire singulière de l'intervention et de l'évolution des relations entre l'interne, le chirurgien et la situation (toujours en termes d'interactions et de contenus). On repère par exemple le nombre d'insertions cutanées nécessaires à la recherche de la position finale de la broche, ou bien la présence plus marquée d'un type d'interaction, comme l'observation en dernière partie d'intervention dans le protocole VSI-1.

- L'étude transversale permet d'étudier les évolutions des épisodes au travers les différentes interventions, pour dégager des éléments qui persistent au delà des singularités de chaque protocole. On repère par exemple que l'autonomie de l'interne est plus présente lors du deuxième temps de la poussée au moteur que lors de son amorce.

Ces traitements nous conduisent à proposer des résultats qualitatifs (description des interactions, de leurs contenus, et analyse en termes de contrat et de processus de validation), accompagnés d'indications quantitatives. En effet, le dénombrement des interactions de chaque type et des éléments qui les constituent (actions, critères de contrôle, prise d'informations, jugements), nous permet de rendre compte de fréquences d'apparition.

En complément de la description de cette démarche, nous faisons ci-dessous trois remarques facilitant la compréhension des traitements qui sont présentés dans la prochaine section.

Tout d'abord, dans les verbalisations certaines actions sont nommées "contrôles » par les acteurs. Ce sont les prises de radio (« contrôle ta position»), mais qui sont bien des actions, nécessitant d'être couplées à une activité de contrôle qui s'appuie sur des critères relatifs à la radio elle-même (« sur l'inlet ») et à la position de la broche sur cette radio («au-dessus des trous »), et sur des informations de la situation à prélever et traiter (visualisation du bord antérieur du sacrum et de l'articulation ilio-sacrée ; visualisation des trous sacrés et de la broche sur la radio). Ajoutons que l'expression «feu », que nous rencontrerons dans plusieurs extraits, est une injonction à prendre une radio. Elle est utilisée lorsque l'appareil de radio est déjà dans la position de l'incidence souhaitée. Dans le cas contraire, le chirurgien nomme la radio souhaitée (« inlet», par exemple).

Ensuite, une difficulté récurrente de l'analyse de données réside dans l'interprétation qu'il est indispensable de faire de certains comportements, qu'ils soient verbaux ou non verbaux. En particulier certaines rétroactions ne sont ni visibles ni audibles, mais nous pouvons parfois les inférer par ce qui suit (une poursuite de l'action indique la validation de l'étape précédente, par exemple). Dans ce cas, nous le signalons par un codage en italique. Dans l'extrait ci-dessous, l'injonction " pan pan » (intervention 144) qui invite l'interne à fixer la broche au maillet dans l'os est de fait une validation de la position de la broche sur la radio de profil qui vient d'être prise. C'est ainsi une rétroaction de type $\mathrm{b}$ (du chirurgien vers l'interne) qui est inférée ici :

VSI-5, Insertion cutanée

\begin{tabular}{|l|l|l|l|l|}
\hline 142 & Ch à MR : feu & 4 & Action & Injonction \\
\hline 143 & [radio profil] & & & \\
\hline 144 & $\begin{array}{l}\text { Ch [donne le maillet } \\
\text { à I] : pan pan }\end{array}$ & $b$ & Action & Injonction \\
\hline
\end{tabular}

Enfin, les épisodes ne sont pas mutuellement exclusifs : certains éléments d'un épisode peuvent être intégrés à un autre, et y prendre un autre statut. Ce n'est qu'à cette condition que nous pouvons identifier des interactions cohérentes. Souvent, la conclusion de l'épisode $\mathrm{n}$ devient le premier élément de l'épisode $\mathrm{n}+1$. Dans l'épisode qui précède nous avons vu que l'intervention 144 est une rétroaction non dite, du chirurgien pour l'interne $(b)$. Elle devient dans l'épisode suivant, ci-dessous, une injonction pour l'interne à fixer la broche :

VSI-5, Fixation

\begin{tabular}{|l|l|l|l|l|}
\hline 144 & $\begin{array}{l}\text { Ch [donne le maillet } \\
\text { à I] : pan pan }\end{array}$ & 5 & Action & Injonction \\
\hline 145 & $\begin{array}{l}\text { [I donne des coups } \\
\text { de maillet }]\end{array}$ & 1 & Action & \\
\hline
\end{tabular}

\section{RÉSULTATS}

\section{Analyse des interactions}

Notre démarche d'analyse nous permet d'identifier des invariances au sein de la singularité des situations, des sujets et de leurs relations, et de proposer une caractérisation des interactions servant la formation. Le dénombrement des interactions nous indique que l'action guidée ( $25 \%)$, l'observation 
( 34 \%) et l'action autonome ( 22\%) apparaissent de façon dominante.

Nous précisons ci-dessous pour chaque type d'interaction identifié, leurs enjeux (travail ou formation) et les contenus qu'ils portent (action, contrôle, information ou jugement). Nous en donnons également une analyse en termes de contrats, et de processus de validation éventuellement engagés.

\section{L'action guidée}

Le schéma général de l'action guidée est de type 5-1: le chirurgien transmet oralement les actions à mener, sous forme d'injonctions que l'interne exécute : "vas-y, pique ta broche ", "va jusqu'à l'os », " pousse », " prends un profil », " vérifie ta position », etc. C'est le cas par exemple de l'épisode présenté précédemment («VSI-5, Fixation »).

Figure 6 - Action guidée, 5-1

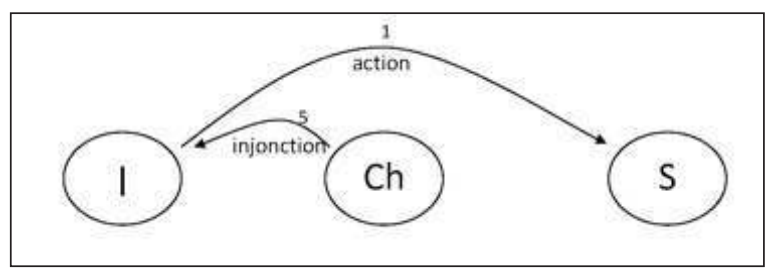

L'action guidée comporte ensuite plusieurs nuances dans la manière dont le chirurgien et l'interne gèrent les rétroactions. Dans la plupart des cas, c'est le chirurgien qui s'en charge, en les verbalisant pour l'interne : "stop, c'est bon », « pas mal », « elle est bien là », " trop bas, tu es trop bas », etc. Le schéma est alors : 5-1-b.

Figure 7 - Action guidée et rétroaction médiée, 5-1-b

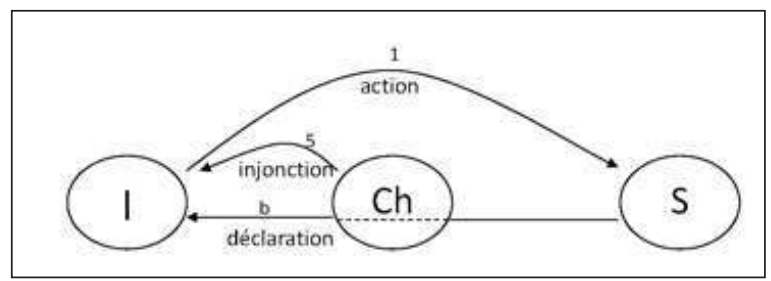

VSI-1, Contrôle face

\begin{tabular}{|l|l|l|l|l|}
\hline 35 & $\begin{array}{l}\text { Ch : tu regardes si } \\
\text { c'est bon }\end{array}$ & 5 & Action & Injonction \\
\hline 36 & I : feu ! & 1 & Action & \\
\hline 37 & [radio face] & & & Déclaration \\
\hline 38 & $\begin{array}{l}\text { Ch }: \text { tu vois, beau- } \\
\text { coup trop haut }\end{array}$ & b & Jugement & Dén \\
\hline
\end{tabular}

Il arrive également que le retour soit fourni par omission, c'est-à-dire que la verbalisation suivante, de nouveau de type 5 - injonction, vienne de fait valider la précédente, mais sans que cette validation ne soit énoncée. Nous avons codé 5-1-b ces interactions :

VSI-2, Fixation

\begin{tabular}{|l|l|l|l|l|}
\hline 20 & $\begin{array}{l}\text { Ch : donne un petit } \\
\text { coup de maillet }\end{array}$ & 5 & Action & Injonction \\
\hline 21 & $\begin{array}{l}\text { [I donne quelques } \\
\text { coups de maillet }]\end{array}$ & 1 & Action & \\
\hline 22 & Ch à MR : feu & $b$ & Action & Injonction \\
\hline
\end{tabular}

Nous observons également, mais plus rarement, des verbalisations conjointes de rétroactions (codées 5-1-a\&b), et des prises en charge autonomes par l'interne, de manière explicite (5-1-a) ou sans énonciation, en poursuivant l'action (5-1-a).

Enfin, un cas particulier de l'action guidée apparaît lorsque l'injonction est donnée sous la forme d'une réponse du chirurgien à un questionnement, direct ou indirect, de l'interne :

VSI-4 : Modification position broche

\begin{tabular}{|l|l|l|l|l|}
\hline 154 & $\begin{array}{l}\text { I : je vais me mettre } \\
\text { légèrement plus en } \\
\text { avant }\end{array}$ & 3 & $\begin{array}{l}\text { Action } \\
\text { Contrôle }\end{array}$ & Déclaration \\
\hline 155 & $\begin{array}{l}\text { Ch: [avec un geste } \\
\text { de la main qui } \\
\text { montre la direction } \\
\text { de la tête du patient] } \\
\text { plus en haut, plus } \\
\text { en avant }\end{array}$ & c & Contrôle & Injonction \\
\hline 156 & $\begin{array}{l}\text { [I repositionne sa } \\
\text { broche] }\end{array}$ & 1 & Action & \\
\hline
\end{tabular}

Au sein des interactions de type action guidée, les verbalisations du chirurgien sont pour plus de la moitié ( $55 \%$ ) des actions à mener (" avance ", «pousse », « prends une radio »), pour $23 \%$ des critères de contrôle (" jusqu'à l'os », " à l'aplomb des lignes ») et pour $16 \%$ des jugements (« c'est 
bon là », « elle est bien »). Plus rarement (6 \%) sont mentionnées les informations à prélever pour rendre opératoires les critères de contrôle. Il semble que les verbalisations sont ajustées à la réalisation de l'action efficace par l'interne. Ainsi, même si l'enjeu de formation est présent par la délégation même de l'acte chirurgical, on détecte dans le minimalisme des interactions verbales et gestuelles l'enjeu de travail.

L'action guidée semble représenter un état d'équilibre entre enjeux de formation et de travail. Les deux protagonistes sont engagés dans un contrat qui n'est pas spécifique de l'intervention observée : on retrouve là des éléments de la coutume que décrit Balacheff (1988). L'interne n'y est ni très sollicité, ni laissé de côté. Le chirurgien lui communique les actions à exécuter, et s'attend à ce qu'il sache les mener. En d'autres termes, le chirurgien prend en charge les décisions d'action, ainsi que les contrôles attenants. Ces derniers relèvent de la vérification, la preuve n'ayant effectivement pas lieu d'être débattue, puisque le chirurgien décide seul du déroulement de l'activité. L'interne n'est pas attendu sur ces aspects, son rôle est celui d'exécutant.

\section{L'action supervisée : le recueil indirect de signes}

L'action supervisée est une interaction que nous n'avions pas définie a priori, composée d'une action guidée et d'un questionnement. Elle apparaît peu ( 8 fois sur les 300 interactions identifiées), mais présente un intérêt particulier quant à l'explicitation de l'activité de contrôle. Ici le chirurgien, en plus de l'action guidée (de type 5-1), questionne l'interne (interaction de type 5-e) sur des informations de la situation qu'il ne peut pas prélever lui-même, à savoir les ressentis au passage de la broche: les signes proprioceptifs (de Montmollin, 1974, p. 36).

Figure 8-Action supervisée, 5-1-5-e



De cette manière, le chirurgien permet aux deux enjeux de travail et de formation de coexister : en obtenant ces informations pour le contrôle il peut ainsi laisser agir l'interne tout en sécurisant cette délégation d'acte, acte dont il reste responsable.

VSI-5, Poussée $1^{\text {re }}$ phase

\begin{tabular}{|c|c|c|c|c|}
\hline 178 & $\begin{array}{l}\mathrm{Ch}: \text { allez / } \\
\text { moteur !/ }\end{array}$ & 5 & Action & Injonction \\
\hline & $\ldots$ & & & \\
\hline 183 & $\begin{array}{l}\text { [I enfonce la } \\
\text { broche au moteur] }\end{array}$ & 1 & Action & \\
\hline 184 & $\begin{array}{l}\text { Ch à I : tu sens que } \\
\text { ça résiste? }\end{array}$ & 5 & Information & Interrogation \\
\hline 185 & $\begin{array}{l}\text { I : pour l'instant ça } \\
\text { résiste }\end{array}$ & e & Information & Déclaration \\
\hline 186 & $\begin{array}{l}\text { [I continue à pous- } \\
\text { ser au moteur / le } \\
\text { son devient plus } \\
\text { aigu] }\end{array}$ & 1 & Action & \\
\hline 187 & $\begin{array}{l}\text { Ch à I : et là ça } \\
\text { résiste plus ? [ø et } \\
\text { non pas +] }\end{array}$ & 5 & Information & Interrogation \\
\hline 188 & $\begin{array}{l}\text { I : ça résiste } \\
\text { toujours }\end{array}$ & $\mathrm{e}$ & Information & Déclaration \\
\hline 189 & Ch à MR : feu & $b$ & Action & Injonction \\
\hline
\end{tabular}

L'action supervisée est suivie (ci-dessous) par une prise de radio demandée par le chirurgien, qui lui permet de compléter sa prise d'informations sur la situation, et ainsi sa compréhension de la position de la broche dans le volume osseux du patient :

VSI-5, Contrôle outlet

\begin{tabular}{|l|l|l|l|l|}
\hline 189 & Ch à MR : feu & 4 & Action & Injonction \\
\hline 190 & [radio outlet] & & & \\
\hline 191 & $\begin{array}{l}\text { Ch à I : c'est parce } \\
\text { que tu viens de } \\
\text { passer l'articulation } \\
\text { sacro-iliaque }\end{array}$ & $b$ & Contrôle & Déclaration \\
\hline
\end{tabular}

Ce suivi de l'action qu'effectue le chirurgien, partant d'une nécessité de l'enjeu de travail (il permet au chirurgien de garantir la non dangerosité de l'acte), a un rôle par rapport à l'enjeu de formation puisqu'il permet à l'interne de prendre connaissance des informations (ici haptiques et sonores) qu'il doit détecter, discriminer et interpréter. Ainsi dans ces phases d'action supervisée l'enjeu de formation, même s'il est secondaire pour le chirurgien par rapport à l'enjeu de travail, se nourrit de ce dernier qui nécessite des verbalisations de la part du chirur- 
gien, parce que l'acte est en partie mené par l'interne : la délégation de l'acte entraîne de fait une modification de l'activité qui fait que l'enjeu de formation est servi par celui de travail.

Les verbalisations qui constituent les épisodes d'action supervisée (5-1-5-e) sont principalement des actions dans la partie 5-1, et des informations dans la partie 5-e. Les informations sont donc bien plus présentes dans ce type d'interaction qu'elles ne le sont dans l'action guidée, décrite précédemment. En fait, nous assistons dans les phases d'action supervisée à une évolution du contrat. Par le questionnement, le chirurgien effectue une surveillance plus étroite que lors de l'action guidée, mais il semble clair pour les deux acteurs que ce sont les particularités de la situation qui la rendent nécessaire : la dangerosité potentielle et des informations difficilement verbalisables, relatives aux sensations perçues. Ainsi on assiste à l'instauration d'un contrat didactique particulier, local et temporaire, au sein de la coutume, au cours duquel l'interne se prête au jeu d'un questionnement presque inquisiteur de la part du chirurgien. Comme dans l'action guidée, le processus de contrôle engagé est de l'ordre de la vérification et non de la preuve : le discours ne se détache pas du cas particulier qui est en train de se dérouler.

\section{L'action autonome de l'interne}

L'action autonome de la part de l'interne se rencontre surtout lors de la prise de radios. On la rencontre également en toute fin d'intervention, lorsque la difficulté est passée, et que l'objectif sanitaire est atteint (dans la $2^{\mathrm{e}}$ phase de la poussée au moteur). Nous observons que le chirurgien ne s'oppose jamais à ce que l'interne demande ou prenne une radio : en termes de contrôles radios, plutôt trop que pas assez semble être la règle, tout en cherchant à minimiser la quantité d'irradiation du patient et de l'équipe chirurgicale. Cependant, l'interprétation des radios est le plus souvent prise en charge par le chirurgien, ce qui donne une interaction de type 1-b :
Figure 9 - Action autonome et rétroaction médiée, 1-b

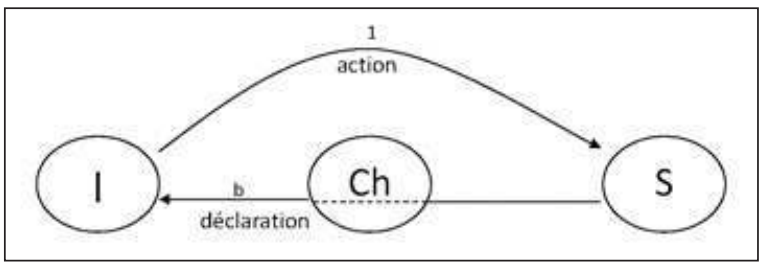

Mais elle est parfois prise en charge par l'interne, verbalisée ou non. S'il y a verbalisation, nous considérons que c'est un questionnement indirect à destination du chirurgien, et nous codons alors 3 cette verbalisation, comme dans l'exemple qui suit.

VSI-3, Contrôle profil : la lecture de la radio est faite sous forme de questionnement indirect de l'interne pour le chirurgien

\begin{tabular}{|l|l|l|l|l|}
\hline 34 & I à MR : feu & 1 & Action & Injonction \\
\hline 35 & [radio profil] & & & \\
\hline 36 & I : donc là je suis... & 3 & & Déclaration \\
\hline 37 & $\begin{array}{l}\text { Ch : trop bas, tu es } \\
\text { trop bas }\end{array}$ & c & $\begin{array}{l}\text { Jugement } \\
\text { Contrôle }\end{array}$ & Déclaration \\
\hline 38 & I : trop bas & 3 & $\begin{array}{l}\text { Jugement } \\
\text { Contrôle }\end{array}$ & Déclaration \\
\hline 39 & Ch : oui & c & & Déclaration \\
\hline
\end{tabular}

S'il n'y a pas verbalisation, l'interaction est codée 1-a, action autonome :

VSI-5, Contrôle face : lors de la $2^{\mathrm{e}}$ phase de poussée au moteur, l'interne prend en charge la prise de radio et son interprétation pour contrôler le point d'arrêt de la broche

\begin{tabular}{|l|l|l|l|l|}
\hline 249 & I à MR : feu & 1 & Action & Injonction \\
\hline 250 & [radio face] & & & \\
\hline 251 & $\begin{array}{l}\text { [I pousse la broche } \\
\text { au moteur] }\end{array}$ & $a$ & Action & \\
\hline
\end{tabular}

Ce type d'interaction, notamment lorsque celleci porte sur la prise de radios, semble permettre à l'interne d'exercer son autonomie, tant au niveau du geste chirurgical qu'à celui de la communication avec le manipulateur radio. Selon la dimension du travail, ces interactions portent la marque de l'autonomie progressive de l'interne : il sait faire certaines choses, et le chirurgien lui permet de les faire. Mais selon la dimension de la formation, ces épisodes ne sont pas neutres non plus. Ils permettent à l'interne de se placer en "position anticipée de maîtrise » (Delbos et Jorion, 1984, p. 144). On le remarque en 
particulier lors des épisodes de réglages de l'appareil de radios, dans la communication de l'interne avec le manipulateur de radio, où tout se passe comme si l'interne était responsable de l'intervention. Dans l'extrait suivant l'évolution du vouvoiement au tutoiement semble être une marque supplémentaire de ce phénomène.

VSI-5, Contrôle profil

\begin{tabular}{|c|c|c|c|c|}
\hline 204 & I à MR : profil & 1 & Action & Injonction \\
\hline 206 & $\begin{array}{l}\text { I à MR : avancez } \\
\text { vers moi, encore } \\
\text { encore encore, ça } \\
\text { passe, encore, bais- } \\
\text { sez, baissez, encore, } \\
\text { bloquez, allez-y, feu, } \\
\text { feu }\end{array}$ & $a \mathrm{l}$ & $\begin{array}{l}\text { A c t i o n } \\
\text { Contrôle }\end{array}$ & Injonction \\
\hline 207 & [radio profil] & & & \\
\hline 208 & $\begin{array}{l}\text { I à MR : euh, retire } \\
\text { un peu vers toi }\end{array}$ & $a 1$ & $\begin{array}{l}\text { A c t i on } \\
\text { Contrôle }\end{array}$ & Injonction \\
\hline 209 & $\begin{array}{l}\text { I à MR : voilà, vas-y } \\
\text { feu }\end{array}$ & $a \mathrm{l}$ & Action & Injonction \\
\hline 210 & [radio profil] & & & \\
\hline
\end{tabular}

$\mathrm{Au}$ cours de certaines phases moins dangereuses de l'intervention le contrat peut également localement évoluer vers une autonomie plus grande pour l'interne. Cette autonomie est contrôlée par le chirurgien, plus ou moins étroitement. Du point de vue de l'interne cependant, l'autonomie le place en phase adidactique, au cours de laquelle c'est à lui de décider des actions à mener, de les exécuter et de les contrôler. Et cette position est plus ou moins tenable par l'interne. Un de nos protocoles (VSI-1) montre l'interne menant l'activité au ralenti, répétant plusieurs fois le même geste, comme à vide, sans faire évoluer la situation, jusqu'à ce que le chirurgien revienne vers lui, marquant la fin du contrat temporairement mis en place et le retour à la coutume. Cette situation n'est malheureusement pas bien rendue par la retranscription des verbalisations, aussi nous ne fournissons pas l'extrait correspondant, qui pourtant illustre toute la validité de l'analyse en termes de contrat : au-delà de l'exécution d'un protocole opératoire, il existe bien un engagement mutuel, non-dit, qui régule les interactions et que l'interne ne rompt pas, même s'il se trouve en situation de détresse dans la position que lui impose ce contrat.

\section{L'observation, ou l'action autonome du chirurgien}

Il arrive également que le chirurgien mène de façon autonome une partie de l'intervention. L'interne est alors placé en situation d'observation. Le cas le plus fréquemment rencontré dans nos protocoles est celui de la prise de radio (dans $73 \%$ des cas d'observation relevés). Les rétroactions sont le plus souvent verbalisées par le chirurgien, ce que nous considérons comme étant délibérément orienté vers l'enjeu de formation. Ces interactions sont codées 4-b.

Figure 10 - Observation et rétroaction médiée, 4-b

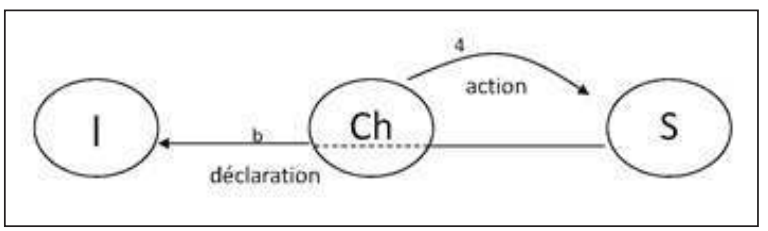

VSI-2, Contrôle inlet

\begin{tabular}{|l|l|l|l|l|}
\hline 43 & Ch à MR : feu & 4 & Action & Injonction \\
\hline 44 & [inlet] & & & \\
\hline 45 & Ch : ok & b & Jugement & Déclaration \\
\hline
\end{tabular}

Par exemple, dans la suite des épisodes ci-dessous (rassemblés pour plus de clarté), le chirurgien ne verbalise pas ce que les radios lui fournissent comme informations sur la position de la broche.

VSI-1, Insertions et contrôles outlet

\begin{tabular}{|l|l|l|l|l|}
\hline 394 & $\begin{array}{l}\text { [Ch modifie la posi- } \\
\text { tion de la broche] }\end{array}$ & 4 & Action & \\
\hline 395 & Ch à MR : feu & 4 & Action & Injonction \\
\hline 396 & [radio outlet] & & & \\
\hline 397 & $\begin{array}{l}\text { [Ch modifie la posi- } \\
\text { tion de la broche] }\end{array}$ & $d 4$ & Action & \\
\hline 398 & Ch à MR : feu & 4 & Action & Injonction \\
\hline 399 & [radio outlet] & & & \\
\hline 400 & $\begin{array}{l}\text { [Ch modifie la posi- } \\
\text { tion de la broche] }\end{array}$ & $d 4$ & Action & \\
\hline 401 & Ch à MR : feu & 4 & Action & Injonction \\
\hline 402 & [radio outlet] & & Action & \\
\hline 403 & $\begin{array}{l}\text { [Ch modifie la posi- } \\
\text { tion de la broche] }\end{array}$ & $d 4$ & & \\
\hline
\end{tabular}

Comment, dans ces conditions d'observation pour l'interne, maintenir l'enjeu de formation? Nous remarquons à plusieurs reprises que le chirurgien donne des marques de la délégation de l'acte à l'in- 
terne dans son discours, même lorsqu'il l'a momentanément fait cesser dans les faits. Sur une broche qu'il a lui-même insérée et fixée, il dira ainsi à l'interne: « allez pousse-la un petit peu ta broche, là ». Ce procédé semble permettre de préserver l'engagement de l'interne dans la situation, c'est une sorte de transaction entre le chirurgien et l'interne qui aboutit au rétablissement du contrat initial, de la coutume, après cette interruption. Nous manquons cependant d'une analyse du discours plus poussée pour étudier ces nuances syntaxiques.

L'observation commentée nous paraît, comme l'action guidée, représenter la coutume en vigueur au bloc opératoire, permettant d'équilibrer les enjeux de travail et de formation. En revanche, lorsque le chirurgien reprend momentanément la main sur l'intervention, comme s'il était seul, ne formulant des phrases qu'à destination du manipulateur radio ou de l'anesthésiste, nous identifions des ruptures de contrat, puisque la formation n'intervient plus dans l'activité.

\section{L'action médiée}

L'action médiée, que nous pensions a priori très présente, est rare dans nos protocoles. Elle n'y apparaît que trois fois, en tout début d'intervention, lors de l'insertion initiale de la première broche: le chirurgien prend la broche avec l'interne et accompagne le geste.

Figure 11 - Action médiée, 2



VSI-1, Insertion

\begin{tabular}{|l|l|l|l|l|}
\hline 1 & $\begin{array}{l}\text { [I a la broche en main } \\
\text { et Ch le guide en } \\
\text { posant ses mains sur } \\
\text { les siennes] }\end{array}$ & 2 & $\begin{array}{l}\text { Action } \\
\text { Contrôle }\end{array}$ & \\
\hline
\end{tabular}

Nous pouvons envisager plusieurs rôles de l'action médiée, liés aux places respectives que peuvent prendre les enjeux de travail et de formation. Sous l'hypothèse d'un enjeu de travail prédominant, nous interprétons ce geste médié comme étant celui du professionnel s'assurant que le point de départ de l'activité n'est pas trop éloigné de ce qu'il devrait être pour obtenir une solution correcte dans des délais raisonnables. Sous l'hypothèse d'un enjeu de formation prédominant, nous pouvons considérer cette action médiée au niveau non plus de l'action mais du contrôle : le chirurgien fournit par l'intermédiaire de son geste les critères de contrôle et les signes relatifs au positionnement et à l'orientation de la broche. Il s'économise peut-être ainsi une verbalisation qu'il sait de toute façon trop imprécise (« la broche doit être orientée de manière un peu ascendante et antérieure »). On perçoit ici la particularité pour la formation de la nature empirique de l'activité.

\section{Le questionnement}

Il arrive qu'apparaissent au sein de l'intervention des questionnements, de type 3-c lorsque c'est l'interne qui questionne le chirurgien. Du point de vue du contenu, plusieurs niveaux d'échanges sont observés, de la simple confirmation de l'action à mener à l'explicitation de critères de contrôle et de prises d'information associées.

VSI-5, Contrôle inlet : un questionnement direct

\begin{tabular}{|l|l|l|l|l|}
\hline 197 & I : inlet ? & 3 & Action & Interrogation \\
\hline 198 & $\begin{array}{l}\text { Ch à I : ouais ouais } \\
\text { c'est bien, oui oui } \\
\text { t'as raison, à mi } \\
\text { chemin faut faire un } \\
\text { contrôle. }\end{array}$ & $\begin{array}{l}\text { A c ti on } \\
\text { Contrôle }\end{array}$ & Déclaration \\
\hline
\end{tabular}

VSI-2, Contrôle profil : un questionnement indirect

\begin{tabular}{|c|c|c|c|c|}
\hline 104 & $\begin{array}{l}\text { I : je suis peut- } \\
\text { etre un peu trop } \\
\text { bas }\end{array}$ & 3 & Jugement & Déclaration \\
\hline 105 & $\begin{array}{l}\text { Ch : t'es arrivé } \\
\text { à la moitié de ta } \\
\text { distance }\end{array}$ & c & Contrôle & Déclaration \\
\hline 106 & I : oui & 3 & & Déclaration \\
\hline 107 & $\begin{array}{l}\text { Ch : or tu restes } \\
\text { en arrière de tes } \\
\text { deux lignes, là }\end{array}$ & c & $\begin{array}{l}\text { Contrôle } \\
\text { Information }\end{array}$ & Déclaration \\
\hline 108 & $\begin{array}{l}\text { Ch : tu vois les } \\
\text { deux lignes? }\end{array}$ & $\mathrm{c}$ & Information & Interrogation \\
\hline 109 & I : oui & 3 & & Déclaration \\
\hline 110 & $\begin{array}{l}\text { Ch : donc ça } \\
\text { veut dire que tu } \\
\text { es parfait }\end{array}$ & $\mathrm{c}$ & Jugement & Déclaration \\
\hline
\end{tabular}


Parfois c'est le chirurgien qui questionne l'interne (5-e). Lors de l'action supervisée (décrite plus haut) nous y avons détecté un enjeu de travail prédominant. Mais il arrive également que le questionnement soit lié à un enjeu de formation, et dans ce cas l'interaction porte sur des prises d'informations nécessaires à l'application des critères de contrôles.

VSI-5, Contrôle inlet

\begin{tabular}{|l|l|l|l|l|}
\hline 172 & $\begin{array}{l}\text { Ch : repère } \\
\text { bien le décro- } \\
\text { chage, t'as vu le } \\
\text { décrochage? }\end{array}$ & 5 & Information & Interrogation \\
\hline 173 & $\begin{array}{l}\text { I : il est là [geste } \\
\text { désignant la } \\
\text { radio] }\end{array}$ & e & Information & Déclaration \\
\hline
\end{tabular}

Le questionnement est une interaction riche du point de vue de l'explicitation des critères de contrôle et des prises d'informations. En particulier celui qui est initié par le chirurgien est le plus porteur des signes du travail. Cependant, on note également qu'il n'est pas très présent dans nos protocoles. On se demande alors, au vu de la rareté des questions posées par l'interne au chirurgien, si le simulacre d'expertise que nécessite la situation de la part de l'interne (qui se doit de jouer le jeu) ne réduit pas dans une certaine mesure les possibilités qu'il se donne de questionner le chirurgien, interaction qui semble pourtant propice à l'explicitation de la structure de contrôle.

Le questionnement apparaît comme une interaction qui n'est pas accompagnée d'une action, mais qui porte sur l'activité. En cela, elle représente une interaction qui peut se combiner aux autres, comme nous l'avons vu dans le cas de l'action supervisée où elle se combine avec l'action guidée. Elle peut porter sur l'action, lorsque par exemple l'interne demande une confirmation avant l'exécution (extrait ci-dessus, «VSI-5 Contrôle inlet : questionnement direct »). On remarque que la réponse du chirurgien se détache parfois du cas particulier et revêt alors un caractère plus général : «à mi-chemin faut faire un contrôle ». Lorsque le questionnement est initié par le chirurgien, il porte un enjeu de formation, et semble destiné à vérifier la présence chez l'interne des éléments permettant de prendre une décision soit quant à l'action à mener, soit quant à la validité de l'action menée. Lors du questionnement, on peut dire que l'enjeu de travail semble un moment s'effacer au profit de celui de la formation.

\section{L'explication}

L'action 5, lorsqu'elle est de type déclaratif, est une explication que le chirurgien mène pour l'interne. Elle apparaît rarement dans nos protocoles (6 fois sur les 300 interactions), et elle est bien sûr liée à un enjeu de formation. Chaque fois, l'explication se limite à l'intervention du chirurgien, sans rétroaction visible chez l'interne :

Figure 12 - Explication, 5

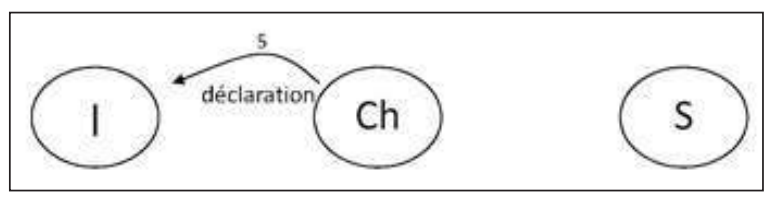

VSI-1, Contrôle inlet

\begin{tabular}{|c|c|c|c|c|}
\hline 213 & $\begin{array}{l}\text { Ch : parce qu'en } \\
\text { fait ton foramen } \\
\text { faut voir que c'est } \\
\text { un ovale, donc } \\
\text { quand t'es là t'as } \\
\text { l'impression que } \\
\text { t'es bien mais si } \\
\text { t'es là et en haut, } \\
\text { si tu es en arrière } \\
\text { et en bas, comme } \\
\text { c'est ovale tu vas } \\
\text { sortir, alors que } \\
\text { quand tu es en bas } \\
\text { et en dessous c'est } \\
\text { bon }\end{array}$ & 5 & $\begin{array}{l}\text { Contrôle } \\
\text { Information } \\
\text { Jugement }\end{array}$ & Déclaration \\
\hline
\end{tabular}

Ces interactions contiennent l'explicitation de critères de contrôle et de signes. De même que pour le questionnement initié par le chirurgien (de type 5-e), l'explication (5 déclaratif) porte un enjeu de formation, et celui de travail semble momentanément absent. Les déclarations prennent dans l'explication une valeur plus générale que dans le questionnement, le discours se détache de l'action particulière qui lui donne naissance, même s'il s'appuie sur les éléments présents. En cela, on peut identifier une évolution de la vérification vers la preuve (Balacheff, 1987), du singulier vers le général (Mondada, 2002). 


\section{Synthèse de l'analyse des interactions}

Au terme de ces analyses, il apparaît que l'observation commentée et l'action guidée sont les deux états d'équilibre entre enjeu de formation et enjeu de travail, équilibres qui définissent la coutume didactique du bloc opératoire. Ces deux types d'interaction sont porteurs d'actions, de critères de contrôles, de jugements, mais peu de signes du travail. L'action guidée fournit en premier lieu à l'interne une suite d'actions : "pique la broche», "pousse », « vérifie », etc. Le caractère en partie répétitif de l'acte chirurgical que nous avons étudié introduit au sein d'une même situation des répétitions qui permettent de percevoir des invariances : si les actions à mener restent attachées à un état particulier de la situation, au hic et nunc, certaines sont répétées à chaque insertion de broche et prennent ainsi un statut qui se démarque du singulier, acquérant une relative généralité. Cependant, nous avons déjà soulevé plus haut le fait que « l'imitation formelle du geste ne garantit pas contre son usage déplacé » (Clot et al., 2007, p. 111), c'est-à-dire que la connaissance de la suite d'actions à mener ne suffit pas à conduire l'activité. Cette idée a fondé la distinction faite en analyse du travail entre tâche et activité (Leplat, 1992) et nous renvoie au modèle de l'activité que nous avons développé plus haut : les actions constituent la partie visible de l'activité, mais sont générées par les autres composantes, qui leur confèrent leur caractère adaptatif. Nous avons constaté que l'action guidée fournit en partie ces autres composantes : des critères de contrôle liés aux actions ("pousse jusqu'à l'os ", " fixe pour que ça ne bouge plus », etc.), des critères de prise de décision de la mise en ouvre des actions («fais un profil à mi-course », « fais un contrôle à l'aplomb des trous », etc.), et quelques signes du travail (sur les radios : «les échancrures », "les lignes »). Pour ce dernier élément, les signes, l'action supervisée (interaction particulière combinant une action guidée et un questionnement) est plus riche, et fournit à l'interne plus d'informations, en particulier sur les ressentis : "ça résiste ", " c'est dur », etc. Enfin, le chirurgien émet au cours de l'action guidée et de l'observation commentée des jugements, qui sont le résultat des processus de validation qu'il engage au cours de l'activité, processus qui sont de l'ordre de la vérification, attachés à la situation singulière.

Certains événements conduisent à des modifications de cette coutume, et ainsi, comme le propose
Balacheff (1988), à l'instauration d'un contrat local, temporaire, avant le retour à la normale. Ainsi l'observation muette, lorsque le chirurgien reprend la main sur l'opération sans même la commenter pour l'interne, ignore momentanément l'enjeu de formation, et par là, rompt la coutume par la mise en place d'un contrat adapté aux nécessités sanitaires de la situation. La fin de ces épisodes est aisément repérable dans les protocoles, par les verbalisations du chirurgien qui invitent l'interne à reprendre sa place dans le déroulement de l'intervention. Nous avons pointé le caractère particulier de ces verbalisations qui paraissent délibérément nier l'épisode de rupture de la coutume. Il semble que ce soit un artifice syntaxique qui permet de recouvrer l'équilibre de la coutume, et qui marque du même coup la fin du contrat local précédemment instauré. De la même manière, mais à l'opposé de l'observation par rapport aux enjeux de travail ou de formation, nous avons identifié l'action autonome. Ici encore la coutume est rompue, et un contrat local s'instaure à l'initiative du chirurgien qui laisse la main à l'interne : « ce changement de contrat didactique est d'une certaine manière une rupture du contrat initial. Le nouveau contrat suppose une autre configuration des transactions didactiques : certaines actions didactiques, jusqu'alors sous la responsabilité du professeur, vont passer dans le territoire de l'élève, qui devra assumer, et pourra ou non le faire " (Sensevy, 2007, p. 19). L'autonomie est une phase adidactique de la formation, qui a pu se mettre en place grâce à l'identification progressive par l'interne des éléments du milieu adidactique. Dans cette identification les verbalisations du chirurgien ont joué un rôle majeur, lors des phases coutumières (fournissant actions, critères de contrôle, jugements), lors des phases d'action supervisée (fournissant des signes), et lors des explications et des questionnements (fournissant critères de contrôle et signes). La fin des phases d'action autonome se caractérise par la vérification, menée par le chirurgien, de l'état d'avancement de l'intervention.

Évoquons pour terminer cette synthèse le statut particulier du questionnement et de l'explication. Nos analyses nous laissent penser que tous deux ne relèvent pas de la négociation d'un contrat local au sein de la coutume, mais qu'ils accompagnent la coutume, permettant parfois de la maintenir (voir par exemple l'épisode "VSI-5 Contrôle inlet : questionnement direct » cité plus haut dans le paragraphe sur le questionnement), parfois de la faire évoluer 
vers des contrats locaux (voir par exemple dans l'épisode «VSI-1 Insertion» cité plus bas dans le paragraphe sur l'erreur : un questionnement qui n'aboutit pas et qui va conduire le chirurgien à reprendre la main). Le questionnement initié par l'interne, mais surtout l'explication, sont riches du point de vue de l'explicitation de critères de contrôle et de signes. On note également une évolution de la vérification vers la preuve au cours de ces interactions, qui se détachent parfois du discours singulier pour traiter de la règle plus générale. Mais nous constatons que même lorsque l'interne le questionne directement, le chirurgien ne considère dans sa réponse que les éléments sur lesquels il pense qu'il pourrait y avoir un doute. En d'autres termes, le non problématique n'est pas rendu visible, et ce qui est non problématique est déterminé par le chirurgien, en tant que professionnel et/ou en tant que formateur. Le reste, ce qu'il considère comme allant de soi pour lui et/ou pour l'interne, n'est pas intégré à ses verbalisations. Une étude plus poussée pourrait nous amener à identifier là des phénomènes de tacitation décrits par Dépret et Maitre (2011), au cours desquels le maître attribue à l'élève - à tort ou à raison - une certaine compréhension de la situation.

Ainsi, les deux enjeux de formation et de travail sont visibles au cours de toutes les interventions étudiées, dans un équilibre qui varie. Le type d'interaction engagée nous permet d'obtenir une première description de ces variations. De l'action guidée, délibérément formative, à l'action autonome du chirurgien où l'enjeu de travail domine, nous avons montré un ensemble de possibles. L'étude systématique des facteurs de cette variation est à poursuivre, mais nous en présentons d'ores et déjà ci-dessous trois aspects : l'erreur, la perte de contrôle et la dangerosité de l'acte.

\section{Facteurs de variations identifiés}

\section{L'erreur}

Étudier le bloc opératoire en tant que système didactique nous amène, en plus de la description des interactions, à nous pencher sur la place de l'erreur dans de telles situations où travail et formation doivent coexister, qui plus est dans un contexte impliquant un patient. De manière quelque peu caricaturale, nous pouvons émettre l'hypothèse que la gestion de l'erreur dans les situations d'internat est un intermédiaire entre le modèle de l'Émile dans la forêt (Rousseau, 1961) et celui du marais (Delbos et Jorion, 1984). Entre une erreur voulue, provoquée, considérée comme vecteur d'apprentissage et une erreur à éviter, une faute dont les conséquences pour la production sont négatives et qu'on sanctionne durement, par « des coups de gueules et des rappels à l'ordre » (ibid., p. 127). Les données étudiées confirment cette hypothèse, mais surtout, permettent de la préciser.

Commençons par une clarification. L'erreur dont nous traitons ici n'est pas celle d'insérer une broche dans une position qui n'est pas retenue au vu des contrôles radios, et qu'il faut retirer. La recherche du bon trajet nécessite la plupart du temps, et ce même pour le chirurgien expert, plusieurs tentatives, et cela n'est pas considéré comme une erreur. Celle-ci en revanche pourra être de corriger une position ou une orientation de la broche dans le mauvais sens, au regard des contrôles radios pris : il s'agit là d'une mauvaise interprétation des radios. L'erreur peut également être de ne pas effectuer tous les contrôles nécessaires (ne pas associer un inlet à un outlet par exemple); ou de mal interpréter les signes, en pensant être à un endroit alors qu'on n'y est pas; ou encore de ne prélever qu'une partie des informations nécessaires (interpréter la position de la broche sur une radio dont on n'a pas vérifié la validité, par exemple). En matière de signes dans cette situation, il semble que la redondance ne soit pas problématique, mais que l'incomplétude le soit. Cela nous ramène à la question de l'apprentissage d'un système cohérent de détection et de traitement des informations chez l'apprenant.

L'identification de l'erreur, en tant que lecture d'une rétroaction de la situation, n'est pas toujours possible pour l'interne. Il se peut qu'il ne perçoive pas les rétroactions négatives de la situation et que le chirurgien soit alors à l'initiative d'une intervention dans sa relation avec la situation. La nature de cette intervention pourra être explicative, et nous associons alors cette intervention à un enjeu de formation, ou injonctive, et nous l'associons plus à un enjeu de travail (rappelons que la réduction du travail à l'enjeu chirurgical est faite ici pour les besoins de l'exposé, mais il est bien entendu que la formation faisant partie du travail du chirurgien, elle n'est pas pertinente en soi).

Dans les deux cas, il est probable que le chirurgien, dans un souci d'efficacité chirurgicale, ne prenne pas les moyens, comme on pourrait le voir en 
classe, d'amener l'interne à comprendre l'inefficacité ou l'inadéquation de son action sur la situation, par rapport à l'objectif. Les prises de parole ou d'instruments peuvent être soudaines, et autoritaires. Nous pourrions dire, en première analyse, que ses interventions sont plus là pour faire agir que pour faire comprendre. Mais de fait, agir et comprendre étant étroitement intriqués au sein du processus de conceptualisation de la situation par l'interne, la distinction n'a pas lieu d'être : l'action guidée permet autant la compréhension que l'observation ou l'explication permettent l'action ultérieure (Piaget, 1974). De ce fait, la gestion d'une situation singulière est toujours en partie la construction d'une généralisation.

Il arrive parfois que l'interne se trouve face à une contradiction. C'est le cas par exemple lorsqu'il pense, fait ou annonce un jugement sur une action effectuée ou bien une anticipation sur l'action suivante à effectuer, et que le chirurgien en fait ou en annonce un autre :

VSI-4, Contrôle profil

\begin{tabular}{|l|l|l|l|l|}
\hline 124 & I : là je suis bon & 3 & Jugement & Déclaration \\
\hline 125 & $\begin{array}{l}\text { Ch : t'es pas bon, tu } \\
\text { vas en plein dans le } \\
\text { canal, pile poil }\end{array}$ & c & $\begin{array}{l}\text { Jugement } \\
\text { Contrôle }\end{array}$ & Déclaration \\
\hline
\end{tabular}

La contradiction n'est rendue possible que par le rôle de médiateur que le chirurgien joue entre la situation et l'interne: on ne pourrait se permettre d'attendre que la situation produise d'elle-même la rétroaction.

Nous pouvons ainsi avancer que l'erreur ne peut pas être considérée comme vecteur d'apprentissage au sens qu'on lui attribue classiquement dans les théories constructivistes sur l'apprentissage. Mais nous voyons apparaître grâce aux interventions de type $b$ du chirurgien, les rétroactions médiées, des potentiels de contradictions qui ont un effet déstabilisant pour l'interne, que nous pouvons rapprocher de celui de l'erreur. En d'autres termes, le chirurgien fait partie du milieu antagoniste (Brousseau, 1998). Ces erreurs sont parfois à l'origine d'une explication de la part du chirurgien :

VSI-1, Contrôle inlet

\begin{tabular}{|l|l|l|l|l|}
\hline 471 & $\begin{array}{l}\text { Ch : fais moi un } \\
\text { inlet, faut être bien } \\
\text { systématique, quand } \\
\text { même, hein, là tu sais } \\
\text { pas où tu es, alors que } \\
\text { t'es déjà vachement } \\
\text { avancé }\end{array}$ & 5 & $\begin{array}{l}\text { A ction } \\
\text { Contrôle }\end{array}$ & Déclaration \\
\hline
\end{tabular}

VSI-3, Poussée $1^{\text {re }}$ phase

\begin{tabular}{|l|l|l|l|l|}
\hline 74 & $\begin{array}{l}\text { I : on ferait pas un } \\
\text { petit outlet? }\end{array}$ & 3 & Action & Interrogation \\
\hline 75 & $\begin{array}{l}\text { Ch : t'as pas dépassé } \\
\text { l'articulation, outlet } \\
\text { c'est quand tu es dans } \\
\text { le sacrum, d'accord }\end{array}$ & c & Contrôle & Déclaration \\
\hline 76 & I : d'accord & 3 & & Déclaration \\
\hline
\end{tabular}

Enfin, nous observons que les erreurs de l'interne provoquent des modifications sur le type d'interaction et la nature des contenus explicités. Ces changements sont-ils les indices d'un équilibre modifié entre enjeux de travail et de formation (le travail devenant prédominant par rapport à la formation), ou est-ce toujours dans un souci de formation que le chirurgien marque l'erreur par un changement dans son attitude, palliant ainsi l'impossibilité qu'il a de laisser la situation montrer d'elle-même à l'interne les conséquences possibles de cette erreur? Nous n'avons pas assez d'éléments pour traiter cette question, qui nécessiterait des investigations supplémentaires, par exemple par entretiens. Dans l'extrait ci-dessous, nous observons que, suite à l'incompréhension de l'interne (à deux reprises, interventions 74 et 76), le chirurgien reprend la broche (à partir de l'intervention 79) tout en expliquant la manière dont il convient de la placer :

VSI-1, Insertion

\begin{tabular}{|c|c|c|c|c|}
\hline 69 & $\begin{array}{l}\text { Ch : alors ce qu'il } \\
\text { faudrait c'est que } \\
\text { tu gardes, que tu } \\
\text { gardes exactement } \\
\text { la broche dans ce } \\
\text { plan là, dans le } \\
\text { même plan }\end{array}$ & 5 & Contrôle & Injonction \\
\hline 70 & $\begin{array}{l}\mathrm{I} \quad \mathrm{m} \mathrm{m} \mathrm{m} \\
\text { [approbation] }\end{array}$ & $\mathrm{e}$ & & \\
\hline 71 & $\begin{array}{l}\mathrm{Ch} \text { : par contre } \\
\text { dans l'autre plan }\end{array}$ & 5 & Contrôle & Injonction \\
\hline 72 & $\begin{array}{l}\text { c'est-à-dire ce plan } \\
\text { là [il illustre par un } \\
\text { geste de la main], }\end{array}$ & 5 & Information & Injonction \\
\hline 73 & $\begin{array}{l}\text { que tu l'amènes } \\
\text { un petit peu plus } \\
\text { haute }\end{array}$ & 5 & $\begin{array}{l}\text { A c t i o n } \\
\text { Contrôle }\end{array}$ & Injonction \\
\hline 74 & $\begin{array}{l}\text { I : donc vers } \\
\text { là-bas? }\end{array}$ & $\mathrm{e}$ & Contrôle & \\
\hline 75 & $\begin{array}{l}\text { Ch: un petit peu } \\
\text { plus haut [répéti- } \\
\text { tion insistante] }\end{array}$ & 5 & Contrôle & Déclaration \\
\hline
\end{tabular}




\begin{tabular}{|c|c|c|c|c|}
\hline 76 & I : comme ça? & $\mathrm{e}$ & & \\
\hline 77 & $\begin{array}{ll}\mathrm{Ch} \quad: \quad \mathrm{n} \text { o n } \\
\text { justement! }\end{array}$ & 5 & & Exclamation \\
\hline 78 & I : ah oui! & $\mathrm{e}$ & & \\
\hline 79 & $\begin{array}{l}\text { Ch : tiens donne- } \\
\text { moi la broche [il } \\
\text { explique ensuite } \\
\text { en montrant avec } \\
\text { la broche en main] }\end{array}$ & 5 & & Injonction \\
\hline 80 & $\begin{array}{l}\text { il faut que } \\
\text { dans le plan } \\
\text { perpendiculaire... }\end{array}$ & 5 & Information & Déclaration \\
\hline 81 & I : là elle est bonne & $\mathrm{e}$ & Jugement & \\
\hline 82 & Ch : elle est bonne, & 5 & Jugement & Déclaration \\
\hline 83 & $\begin{array}{l}\text { donc il faut que tu } \\
\text { te déplaces dans ce } \\
\text { plan là, par contre } \\
\text { dans l'autre plan } \\
\text { il faut que tu te } \\
\text { déplaces comme ça }\end{array}$ & 5 & $\begin{array}{l}\text { A c t i o n } \\
\text { Cont rôle } \\
\text { Information }\end{array}$ & Injonction \\
\hline 86 & $\begin{array}{l}\text { I : vers le haut, } \\
\text { d'accord }\end{array}$ & $\mathrm{e}$ & Contrôle & \\
\hline 87 & $\begin{array}{l}\mathrm{Ch}: \text { donc en fait tu } \\
\text { piques là, exacte- } \\
\text { ment parallèle }\end{array}$ & 5 & $\begin{array}{l}\text { A c t i o n } \\
\text { Contrôle }\end{array}$ & Déclaration \\
\hline 88 & $\begin{array}{l}\text { [Ch pique en même } \\
\text { temps qu'il le dit] }\end{array}$ & 4 & Action & \\
\hline
\end{tabular}

L'enjeu de formation semble clairement pris en charge dans cet échange. Le chirurgien souhaite amener l'interne à mener un raisonnement sur la correspondance entre les radios et le corps du patient. Il finira, après une répétition interrogative en 75 , par une explication verbale et gestuelle - dans laquelle on note la marque de la délégation par l'emploi de la deuxième personne - puis une démonstration du geste : il pique lui-même la broche.

\section{L'avancée du temps chirurgical : un faux indicateur}

De manière générale au bloc opératoire, il est important de minimiser la durée de l'intervention. Il est en effet dommageable pour plusieurs raisons que l'intervention ne dure trop : outre les contraintes organisationnelles que cela peut engendrer dans le service, l'allongement de la durée accroît la fragilisation du patient par l'augmentation du nombre de radios prises et donc de l'irradiation, et par l'augmentation du temps d'anesthésie.

Lors d'une intervention particulièrement longue (VSI-1, 43 minutes), nous pouvions donc nous attendre à un changement d'équilibre entre enjeu de travail et de formation, au détriment de ce dernier, pour finaliser l'intervention au plus vite. Nous observons bien une prise en charge accrue de la situation par le chirurgien en fin d'intervention, de l'action guidée 5-1-b à l'action commentée 4-b, pour une même étape de l'intervention (insertion cutanée de la broche). En revanche, le chirurgien laisse l'interne prendre en charge la deuxième partie de la poussée, qui n'est plus dangereuse. Ainsi si le chirurgien semble chercher à réduire le temps chirurgical en reprenant la main sur l'insertion, ce n'est que sur le temps de recherche du bon trajet qu'il le fait.

Nous observons ainsi que l'enjeu de travail n'efface pas forcément celui de la formation au fur et à mesure que le temps avance. Il ne devient prédominant que lorsque la situation sort de la norme pour entrer dans le problématique, détecté ici par la durée qui devient exagérément longue.

Ainsi, au-delà d'une première interprétation possible reposant sur le temps, nous pouvons considérer que cette durée qui augmente est la conséquence d'un autre facteur, celui de la perte de contrôle du chirurgien sur la situation : au vu de tous les essais infructueux, il ne trouve plus de possibilité de guidage de l'interne. Il reprend alors en charge l'action pour comprendre ce qui gêne la réussite de l'activité. Une fois la situation de nouveau comprise par le chirurgien il redonne la main à l'interne. C'est sur cet indice que nous nous basons pour avancer que ce n'est pas seulement la durée de l'intervention qui a motivé son changement de prise en charge, puisque cette dernière phase progressera de nouveau au rythme de la formation, avec des actions guidées, des actions autonomes et une action supervisée. Ainsi, on voit ici que les modifications locales du contrat sont également pour le chirurgien un moyen de réguler l'enjeu de travail.

\section{La dangerosité de l'acte}

L'équilibre entre enjeux de travail et de formation est également dépendant du caractère plus ou moins dangereux de la phase de l'activité. Nous repérons dans tous nos protocoles une phase de vigilance accrue du chirurgien sur l'action menée par l'interne lors de la première phase de la poussée au moteur, entre l'impact osseux et l'arrivée à mi-course, à l'aplomb des premiers trous sacrés. C'est la phase 
la plus dangereuse car la plus délicate à contrôler : il est difficile de s'assurer que la broche reste intraosseuse car elle doit passer par une zone étroite. Les indices pris sont les radios inlet et outlet, la radio de profil, les variations du son qu'émet le moteur, et les sensations de résistances au passage de la broche, qui viennent compléter les informations fournies par les radios. Tout ceci constitue un système complexe de signes permettant au chirurgien d'accéder à une représentation de la position de sa broche au sein de la structure osseuse du patient. À ces signes peuvent se rajouter certaines particularités de la situation, qui en caractérisent à la fois l'aspect générique (« c'est une disjonction pure »), et l'aspect singulier (« il est petit ce monsieur, alors il a des pièces osseuses petites »).

Durant les phases les plus dangereuses, le chirurgien a besoin de se forger une représentation de l'action en cours, même si ce n'est pas lui qui l'accomplit. On assiste alors à un questionnement du chirurgien, précis et qui peut à certains moments avoir une fréquence élevée, sur les sensations perçues par l'interne. Nous avons qualifié ces phases d'actions supervisées (voir plus haut), elles permettent au chirurgien de suivre l'activité et de garantir ainsi la non dangerosité de l'acte pour le patient.

Une fois cette première phase de poussée au moteur passée et validée, la deuxième phase n'est plus gérée de la même manière. Dans tous nos protocoles nous notons une délégation plus grande de la part du chirurgien, qui laisse parfois l'interne agir de manière autonome (les interactions sont de type 1-a ou 1-b), surveillant toutefois le point d'arrêt de la broche ( tu dépasses la ligne médiane »). La délégation peut même être marquée physiquement, le chirurgien s'écartant de la table d'opération pour aller répondre au téléphone - ce qu'il ne fait jamais auparavant - ou vérifier avec l'instrumentiste le matériel qui servira à la suite de l'intervention.

\section{CONCLUSION, PERSPECTIVES}

Cette étude didactique du bloc opératoire en tant que lieu de travail et de formation a permis d'avancer principalement sur deux aspects.

Tout d'abord, nous avons pu décrire le fonctionnement du bloc opératoire en termes de coutume et de contrat didactiques. Nous avons montré de quelle manière ils permettent au chirurgien de gérer la coexistence des deux enjeux de formation et de travail, dans un équilibre qui varie en fonction de l'évolution de la situation. La coutume, fondée principalement sur l'action guidée et l'observation, est régulatrice du fonctionnement stable des relations entre chirurgien et interne. Elle est liée aux caractéristiques épistémologiques et institutionnelles du domaine : empirie, prise d'expérience et « imitation prestigieuse » (Mauss, 2010) paraissent en être les principaux déterminants. La coutume se trouve parfois rompue par l'instauration d'un contrat plus local et temporaire, qui peut conduire par exemple à des épisodes d'autonomie de l'interne ou au contraire de mise à l'écart. Ce processus de mise en place d'un contrat local s'appuie sur la stabilité de la coutume qui en rend viable les écarts, les renégociations et les ruptures temporaires, et est lié aux caractéristiques particulières de la situation dans laquelle il s'élabore. Ainsi coutume et contrats permettent la viabilité d'une relation didactique au bloc opératoire alors même que certaines des conditions que l'on pourrait penser requises en situation de formation ne sont pas réunies : le chirurgien prend en charge l'essentiel des rétroactions, des jugements sur la situation, et une grande partie des décisions concernant les actions à mener. En effet, il se doit en particulier d'anticiper les rétroactions du milieu qu'il ne peut laisser s'exprimer sans son intermédiaire. Dans le cadre de la théorie des situations didactiques, cette configuration n'est $a$ priori pas propice au succès du jeu de l'élève, au cours duquel le maître ne devrait pas avoir à intervenir directement, laissant le milieu matériel réagir aux actions de l'élève et fournir à celui-ci les rétroactions nécessaires à son évolution (comme dans la situation emblématique du puzzle, par exemple). Or elle est ici, par l'instauration d'une coutume spécifique, porteuse des potentialités de formation. Le contrat quant à lui, plus localement adapté aux conditions singulières de la situation, permet des ajustements qui maintiennent les deux enjeux de travail et de formation tout au long de l'intervention. Notons qu'il apparaît dans ce travail que la coutume est spécifique du contenu, contrairement à ce que supposait Sarrazy (1995) en cherchant les raisons de la désuétude de ce concept en didactique des mathématiques. Nos travaux s'inscrivent ainsi dans la lignée de la didactique comparatiste qui affirme une généricité de certains concepts de la didactique, mais sans pour autant leur ôter le caractère spécifique des savoirs, qui fonde l'étude du système didactique. La coutume et le contrat, repérés 
en classe de mathématiques, se particularisent ici dans les spécificités du bloc opératoire, qui sont notamment la coexistence des enjeux de travail et de formation, la nature compagnonnique de l'institution et la nature empirique de l'activité.

Le deuxième apport de ce travail est d'avoir précisé le fonctionnement et le contenu des interactions qui se déroulent au bloc opératoire, permettant ainsi une meilleure compréhension de la formation. L'observation commentée, l'action guidée, mais également l'autonomie, sont autant d'interactions qui étaient déjà mentionnées dans les études que nous avons citées en première partie de cet article, mais sans pour autant qu'y soient décrites leurs caractéristiques. Nous en avons proposé une description précise, nous en avons identifié les contenus (actions, critères de contrôle, jugements, signes) et les processus de validation qu'elles véhiculent (vérifica- tion ou preuve). Nous pensons que ce travail pourra donner un cadre d'analyse pour d'autres formations en situation de travail. Une étude en cours sur les Compagnons charpentiers nous permettra d'avancer sur cette question (Meurger et Vadcard, 2012).

Enfin, une perspective importante de ce travail est d'étudier les conséquences sur l'interne, au niveau des apprentissages, des interactions repérées et de la nature des éléments transmis. Une telle étude nécessite de déterminer des points de mesure d'une évolution de l'apprenti, et la mise en place de situations de recueils adaptées. L'enjeu serait alors de caractériser les variables des situations qui favorisent chez l'apprenti les possibilités progressives d'élaboration d'invariants opératoires et de généralisations à partir des situations singulières qu'il rencontre au cours de sa formation. Ce travail reste à faire, tant sur les internes que sur les Compagnons charpentiers. 


\section{RÉFÉRENCES}

Albanese, M., \& Mitchell, S. (1993). Problem-based learning : A review of literature on its outcomes and implementation issues. Academic Medicine, 68, 52-81

Artigue, M. (1990). Ingénierie didactique. Recherches en Didactique des Mathématiques, 9(3), 281-308.

Balacheff, N. (1987). Processus de preuve et situations de validation. Educational Studies in Mathematics, 18, 147176.

Balacheff, N. (1988). Le contrat et la coutume - deux registres des interactions didactiques. In C. Laborde (Ed.), Actes du premier colloque franco-allemand de didactique (pp. 15-26). Grenoble : La Pensée Sauvage.

Balacheff, N. (1995). Conception, propriété du système sujet/milieu. In R. Noirfalise \& M.J. Perrin-Glorian (Eds), Actes de la VIIe École d'été de didactique des mathématiques (pp. 215-229). Clermont-Ferrand : IREM.

Barrows, H.S. (1987). Simulated (standardized) patients and other human simulations. Chapel Hill : Health Sciences Consortium.

Barrows H.S., \& Feltovich P. (1987). The clinical reasoning process. Medical Education, 21, 86-91.

Becmeur, F., Grandadam, S., Kirch, M., \& Mutter, D. (2004). Quels moyens de formation en chirurgie ? À propos d'une enquête aux hôpitaux universitaires de Strasbourg auprès des internes en chirurgie, Annales de Chirurgie, 129(8), 405-409.

Billett, S. (2011). Learning in the circumstances of work: the didactics of practice. Education \& Didactique, 5(2), $125-146$

Blavier, A., \& Nyssen, A.S. (2010). Étude de l'impact des nouvelles technologies sur les modes de coopération des chirurgiens par l'analyse des communications sur le terrain. Le Travail Humain, 73(2), 123-140.

Brousseau, G. (1998). Théorie des situations didactiques. Grenoble: La Pensée Sauvage.

Chevallard, Y. (1997). Les savoirs enseignés et leurs formes scolaires de transmission : un point de vue didactique. Skholê, 7, 45-64.

Chevallier, D. (Dir.). (1991). Savoir faire et pouvoir transmettre. Paris : Éditions de la Maison des Sciences de l'Homme.

Clot, Y., Fernandez, G., \& Scheller, L. (2007). Le geste de métier : problèmes de la transmission. Psychologie de l'interaction, 23/24, 109-138.

Cornu, R. (1988). La métis professionnelle. In C. Baudelot \& R. Cornu, Formes de socialisation de la jeunesse populaire et métis professionnelle (Rapport de contrat) (pp. 200-211). Université de Nantes.

Dachez, R. (2008). Histoire de la médecine de l'Antiquité au $\mathrm{xx}^{\mathrm{e}}$ siècle. Paris : Tallandier

de Castéra, B. (1996). Le compagnonnage. Paris : Presses Universitaires de France.

de Keyser V., \& Nyssen A.S. (1993). Les erreurs humaines en anesthésie. Le Travail Humain, 56 (2/3) (« Analyse cognitive du travail : hommage à Jacques Leplat »), 243-266.
Delbos, G., \& Jorion, P. (1984). La transmission des savoirs. Paris : Éditions de la Maison des Sciences de l'Homme. de Montmollin, M. (1974). L'analyse des situations. Préalable à la formation. Paris : Armand Colin Formation.

de Montmollin, M. (1984). Lintelligence de la tâche. Éléments d'ergonomie cognitive. Berne : Peter Lang.

Dépret, C., \& Maitre, J.-P. (2011). Tacite et implicite : une caractérisation des productions langagières didactiques et de leurs enjeux épistémiques. Recherches en Éducation, Hors-série $n^{\circ} 3,66-79$.

Eraut, M. (2004). Informal learning in the workplace. Studies in Continuing Education, 26(2), 247-273.

Eva, K. (2005). What every teacher needs to know about clinical reasoning. Medical Education, 39(1), 98-106.

Guédez, A. (1994). Compagnonnage et Apprentissage. Paris : Presses Universitaires de France.

Hauge, L.S., Wanzek, J.A., \& Godellas, C. (2001). The reliability of an instrument for identifying and quantifying surgeons' teaching in the operating room. American Journal of Surgery, 181(4), 333-337.

Johsua, S. (1997). Le concept de Transposition Didactique peut-il étendre sa portée au-delà de la didactique des sciences et des mathématiques? Skholê, 6, 15-23.

Kolb, D.A. (1984). Experiential Learning : experience as the source of learning and development. Englewood Cliffs, NJ : Prentice Hall.

Latour, B. (1996), Sur la pratique des théoriciens. In J.-M. Barbier (Ed.), Savoirs théoriques et savoirs d'action (pp. 131-146). Paris : Presses Universitaires de France.

Laurençon, M.F. (1988). Apprendre un métier ou former pour un emploi ? Le point de vue des enseignants. In C. Baudelot \& R. Cornu, Formes de socialisation de la jeunesse populaire et métis professionnelle (Rapport de contrat) (pp. 113-146). Université de Nantes.

Le Boterf, G. (2010). Construire les compétences individuelles et collectives : agir et réussir avec compétence. Paris : Eyrolles.

Legoux, Y. (1972). Du compagnon au technicien. Paris : Techniques et vulgarisation.

Leplat, J. (1992). Lanalyse du travail en psychologie ergonomique. Toulouse : Octarès.

Leplat, J. (1995). À propos des compétences incorporées. Éducation Permanente, 123, 101-114.

Lyon, P. (2003). Making the most of learning in the operating theatre : student strategies and curricular initiatives. Medical Education, 37, 680-688.

Lyon, P. (2004). A model of teaching and learning in the operating theatre. Medical Education, 38, 1278-1287.

McCulloch, P., Mishra, A., Handa, A., Dale, T., Hirst, G., $\&$ Catchpole, K. (2009). The effects of aviation-style non-technical skills training on technical performance and outcome in the operating theatre. Quality \& Safety in Health Care, 18, 109-115.

Marescaux, J., Mutter, D., Soler, L., Vix, M., \& Leroy, J. (1999). Luniversité virtuelle appliquée à la téléchirurgie : de la télé-éducation à la télé-manipulation. Chirurgie, 124(3), 232-239. 
Margolinas, C. (1993). De l'importance du vrai et du faux dans la classe de mathématiques. Grenoble: La Pensée Sauvage.

Mauss, M. (2010). Sociologie et anthropologie. Paris : Presses Universitaires de France ( $1^{\text {re }}$ édition 1950).

Mercier, A. (2008), Didactique et Pédagogie. In A. Van Zanten (Dir.), Dictionnaire de l'éducation (pp. 149153). Paris : Presses Universitaires de France.

Mercier, A., Schubauer-Leoni, M.L., \& Sensevy, G. (2002). Vers une didactique comparée. Revue Française de Pédagogie, 141, 5-16.

Meurger, C., \& Vadcard, L. (2012). Une étude didactique des liens entre agir et comprendre au cours de la formation $d u$ compagnon charpentier. Communication présentée à la Biennale Internationale de l'Éducation. Paris. France. Juillet.

Mondada, L. (2002). Opérer et enseigner à opérer - description de l'action et formulation du savoir-faire. Raisons Pratiques ( $N^{\circ}$ spécial La régularité. Habitude, disposition et savoir-faire dans l'explication de l'action, C. Chauviré \& A. Ogien, Eds), 13, 293-318.

Nyssen, A.S., Larbuisson, R., Janssens, M., Pendeville, P., \& Mayné, A. (2002), A comparison of the training value of two types of anesthesia simulators : computer screen-based and mannequin-based simulators. Anesthesia and Analgesia, 94(6), 1560-1565.

Ombredane, A. (1992), Lanalyse du travail - Facteur d'économie humaine et de productivité. In J. Leplat (Coord.), Lanalyse du travail en psychologie ergonomique (pp. 9-22). Toulouse : Octarès.

Pastré, P. (2001). Travail et compétences : un point de vue de didacticien. In J. Leplat \& M. de Montmollin (Eds), Les compétences en ergonomie (pp. 147-160). Toulouse : Octarès.

Pastré, P. (2008). La didactique professionnelle : origines, fondements, perspectives. Travail et Apprentissage, 1, 9-21.

Peneff, J. (1997). Le travail du chirurgien : les opérations à cour ouvert. Sociologie du travail, 3, 265-296.

Peugnet, F., Dubois, P., \& Rouland J.-F. (1998). Virtual reality versus conventional training in retinal photocoagulation : a first clinical assessment. Computer Aided Surgery, 3(1), 20-26.

Piaget, J. (1974). Réussir et comprendre. Paris : Presses Universitaires de France.

Prioreschi, P., \& Babin, D. (1995). Cos et Cnide. Histoire des Sciences Médicales, XXIX (4), 317-324.

Rabier, C. (2010). La disparition du barbier chirurgien. Analyse d'une mutation professionnelle au XVIII ${ }^{\mathrm{e}}$ siècle. Annales. Histoire, Sciences Sociales, 2010/3, 679-711.

Ramé, L., \& Ramé, S. (1995). La formation professionnelle par apprentissage. Paris : L'Harmattan (collection Logiques Sociales).

Reason, J. (1995). Safety in the operating theatre Part 2 : Human error and organisation failure. Current Anaesthesia \& Critical Care, 6(2), 121-126

Reznick, R.K. (2005). Surgical simulation - A vital part of our future. Annals of Surgery, 242(5), 640-641.
Rousseau, J.-J. (1961). Émile ou De l'éducation. Paris : Gar-

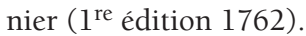

Sakka, M. (1998). La révolution vésalienne, ou l'anatomie devient une science. Les dossiers d'archéologie, 231, 5063.

Sarrazy, B. (1995). Le contrat didactique. Revue Française de Pédagogie, 112, 85-118.

Sensevy, G. (2002). Des catégories pour l'analyse comparée de l'action du professeur : un essai de mise à l'épreuve. In P. Venturini, C Amade-Escot \& A. Terrisse (Coord.), Études des pratiques effectives : l'approche des didactiques (pp. 25-46). Grenoble : La Pensée Sauvage.

Sensevy, G. (2007). Des catégories pour décrire et comprendre l'action didactique. In G. Sensevy \& A. Mercier (dir.), Agir ensemble - L'action didactique conjointe du professeur et des élèves (pp. 13-49). Rennes : Presses Universitaires de Rennes.

Stegmann, K., Zottmann, J., Siebeck, M., \& Fischer, F. (2009). Simulation-based learning : Analysing and fostering complex skills in the context of medical education. Paper presented at the 13th Biennial Conference of the European Association for Research on Learning and Instruction (EARLI). Amsterdam. Pays-Bas. Août.

Testas, P., Mouiel, J., \& Delaitre, B. (1995). L'enseignement de la chirurgie coelioscopique digestive en France : de la formation à l'accréditation ? Bulletin de l'Académie nationale de médecine, 179(7), 1507-1516.

Tonetti, J., Cloppet, O., Clerc, M., Pittet, L., Merloz, P., \& Chirossel, J.-P. (2000). Implantation des vis ilio-sacrées : simulation de l'emplacement optimal. Revue de Chirurgie Orthopédique, 86, 360-369.

Tonetti, J. (2003). Réalisation d'outils de réalité augmentéeApprentissage, simulation et guidage de gestes en chirurgie du bassin (thèse de doctorat non publiée). Université Joseph Fourier, Grenoble 1, France.

Troccaz, J., Henry, D., Laieb, N., Bosson, J.L., \& Pichot, O. (2000). Simulators for medical training : application to vascular ultrasound imaging. Visualization and Computer Animation, 11, 51-65.

Vadcard, L., \& Luengo, V. (2005). Réduire l'écart entre formation théorique et pratique en chirurgie : conception d'un EIAH. In P. Tchounikine, M. Joab \& L. Trouche (Eds.), Actes de la conférence EIAH 2005 (pp. 129-139). Lyon : INRP.

Vadcard, L., de Vries, E., \& Baillé, J. (2009). Authenticité et sémioticité : deux paradoxes de l'expérience dans les technologies informatiques pour la formation. Communication présentée au $1^{\mathrm{er}}$ Colloque international francophone de l'association Recherches et Pratiques en didactique Professionnelle. Dijon. France. Décembre.

Vadcard, L., Tonetti, J., \& Dubois, M. (2011). Développement de l'expérience en chirurgie orthopédique percutanée. Travail et Apprentissages, 6, 77-92.

Vergnaud, G. (1990). Catégories logiques et invariants opératoires. Archives de Psychologie, 58, 145-149.

Vergnaud, G. (1991). La théorie des champs conceptuels. Recherches en Didactique des Mathématiques, 10(2/3), 133-170. 
Vergnaud, G. (1996). Au fond de l'action, la conceptualisation. In J.-M. Barbier (Dir.), Savoirs théoriques et savoirs d'action (pp. 275-292). Paris : Presses Universitaires de France.

Vergnaud, G., \& Récopé, M. (2000). De Revault d'Allonnes à une théorie du schème aujourd'hui. Psychologie Française, 45(1), 35-50.

Vergnaud, G. (2001). Forme opératoire et forme prédicative de la connaissance. In J. Portugais (Ed.), La notion de compétence en enseignement des mathématiques, analyse didactique des effets de son introduction sur les pratiques et sur la formation, Actes du Colloque GDM-2001 (pp. 6-27). Montréal.

Vial, R. (1999). Histoire de l'enseignement des hôpitaux de Paris - Les blouses blanches en formation initiale et continue. Paris : LHarmattan

Wicart, P. (2003). Formation technique des internes en chirurgie (mémoire de M2R non publié). Université Pierre et Marie Curie, Paris, France.

Tableau : Analyse a priori des séances sur l'empire de Charlemagne 